
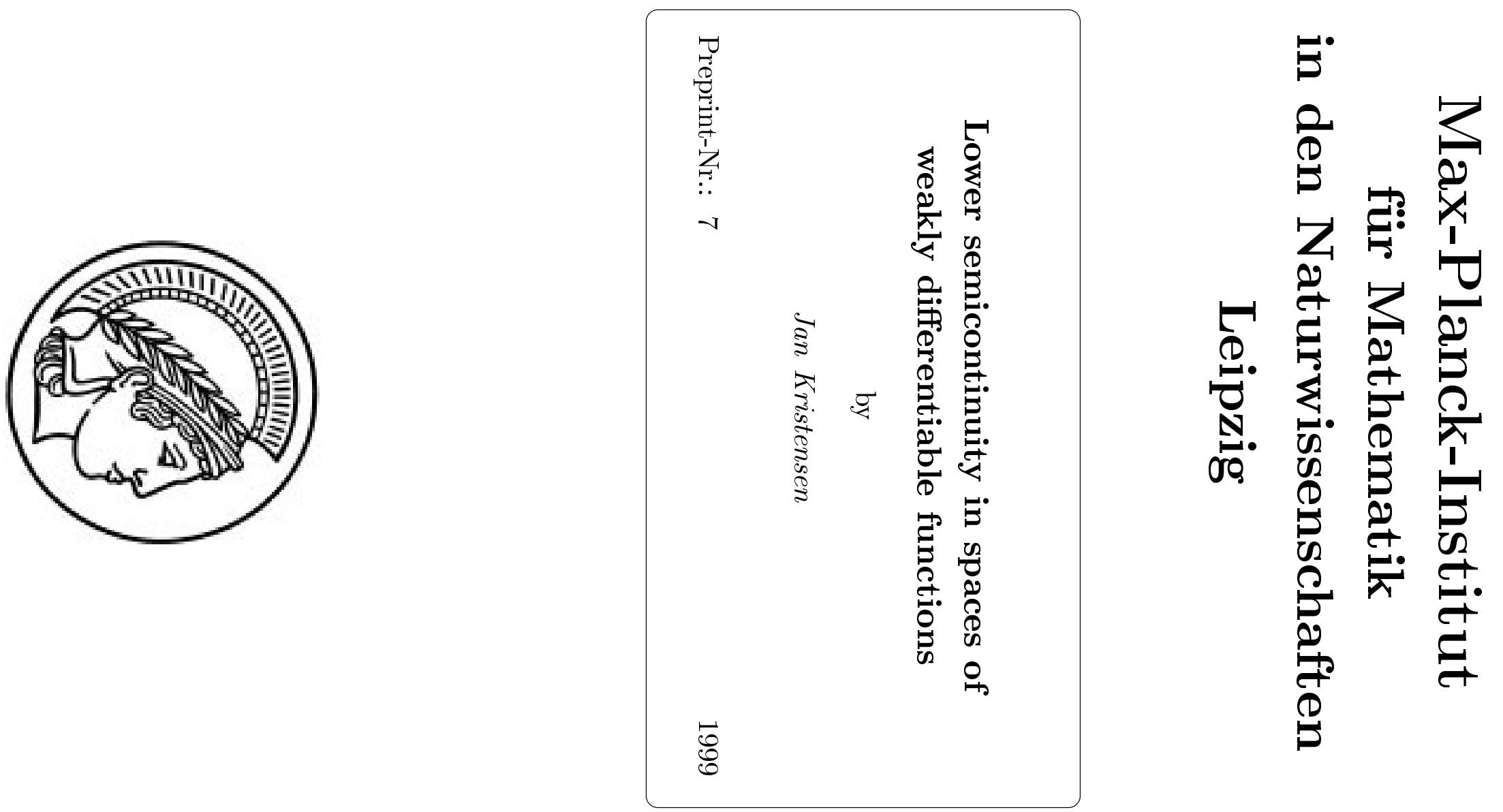



\title{
Lower semicontinuity in spaces of weakly differentiable functions*
}

\author{
Jan Kristensen $^{\dagger}$
}

\section{Introduction and the main results}

In this paper we adopt the approach initiated by Balder in [11] for the study of multiple integrals on Lebesgue spaces, and use Young measures to obtain new lower semicontinuity results for multiple integrals on $B V\left(\Omega ; \mathbf{R}^{n}\right)$, the space of functions of bounded variation. The multiple integrals covered by the results are of the form

$$
I(u)=\int_{\Omega} F(x, u, \nabla u) d x,
$$

where $\Omega \subset \mathbf{R}^{m}$ is an open and bounded set, $F(x, v, X)$ is a normal integrand and $\nabla u$ is the density of the absolutely continuous part (with respect to Lebesgue measure) of the distributional gradient of $u$.

It is well known that the natural convexity assumption in the multi-dimensional calculus of variations is quasiconvexity as introduced by Morrey in [43]. (Notation and definitions are given in Section 2.) The classical lower semicontinuity results for multiple integrals defined on a Sobolev space $W^{1, p}\left(\Omega ; \mathbf{R}^{n}\right)$ (cf. [43], [42], [1] and [40]) state that under suitable growth conditions related to $p$, quasiconvexity is a necessary and sufficient condition for sequential weak lower semicontinuity. Without the growth conditions the lower semicontinuity results fail (cf. [15]). The growth conditions can be relaxed if one adopts the approach proposed in [53]. It amounts to redefining $I(u)$ for non-smooth $u$ by a relaxational procedure and is known as the Lebesgue-Serrin extension of $I(u)$. In the context of quasiconvex integrands this programme was begun in [41] and there is by now numerous papers on the subject. Related to the study undertaken here are, in particular, [9], [24] and [19], where results on lower semicontinuity and relaxation were obtained for the Lebesgue-Serrin extension of multiple integrals defined on $B V\left(\Omega ; \mathbf{R}^{n}\right)$. Without certain growth conditions the relaxational procedure defining the Lebesgue-Serrin extension can fail to provide an extension (cf. [39]). We refer to [21] and [45] for a systematic exposition and further references on quasiconvexity and lower semicontinuity.

Unless otherwise specified we assume throughout the paper that $m, n>1$ and that $\Omega$ is an open and bounded proper subset of $\mathbf{R}^{m}$. We define $I(u)$ for all relevant functions

\footnotetext{
${ }^{*}$ Supported by the Danish Natural Science Research Council through grant no. 9501304 .

${ }^{\dagger}$ Mathematical Institute, University of Oxford, England
} 
by the formula (1.1). The integral in (1.1) is understood as a Lebesgue integral, or if necessary, as an upper Lebesgue integral.

The main results are contained in Theorems 1.1-1.4. However, we believe that some of the auxiliary results may be of independent interest, in particular Lemma 1.7, which contains a result on truncation of sequences of gradients, and Proposition 1.10 on approximation of quasiconvex functions.

Theorem 1.1 pertains to the case where $u$ is of bounded variation and has a distributional gradient $D u$, which is absolutely continuous with respect to a fixed non-negative and finite Radon measure $\mu$. Following [8] we define for each $p \in[1, \infty]$ the space

$$
W_{\mu}^{1, p}\left(\Omega ; \mathbf{R}^{n}\right)=\left\{u \in B V\left(\Omega ; \mathbf{R}^{n}\right): D u \ll \mu, \frac{d D u}{d \mu} \in L_{\mu}^{p}\right\},
$$

where $d D u / d \mu$ denotes the Radon-Nikodym derivative of $D u$ with respect to $\mu$. The Sobolev spaces with respect to a measure enjoy the same compactness properties as the usual Sobolev spaces (cf. [8]). In particular, we note that if $\phi:[0, \infty) \rightarrow[0, \infty]$ is a non-decreasing function, which satisfies the condition

$$
\frac{\phi(t)}{t} \rightarrow \infty \text { as } t \rightarrow \infty
$$

and if $\left\{u_{j}\right\} \subset W_{\mu}^{1,1}\left(\Omega ; \mathbf{R}^{n}\right)$ satisfies

$$
\sup _{j}\left(\int_{\Omega}\left|u_{j}\right| d x+\int_{\Omega} \phi\left(\left|\frac{d D u_{j}}{d \mu}\right|\right) d \mu\right)<\infty,
$$

then for some subsequence of $\left\{u_{j}\right\}$ (for convenience not relabelled) and some $u \in W_{\mu}^{1,1}\left(\Omega ; \mathbf{R}^{n}\right)$

$$
u_{j} \rightarrow u \text { strongly in } L_{l o c}^{1} \text { and } \frac{d D u_{j}}{d \mu} \rightarrow \frac{d D u}{d \mu} \text { weakly in } L_{\mu}^{1} \text {. }
$$

Definition Let $p \in[1, \infty]$. An integrand $F=F(x, v, X): \Omega \times \mathbf{R}^{n} \times \mathbf{R}^{n \times m} \rightarrow \mathbf{R}$ belongs to the class $\mathcal{I}_{p}$ if it satisfies the following three conditions:

(H1) $F=F(x, v, X)$ is a normal integrand, i.e., Borel measurable and lower semicontinuous in $(v, X)$.

(H2) $F(x, v, X)$ is quasiconvex in $X$ for almost all $x$ and all $v$.

$\left(\mathrm{H} 3_{p}\right)$ For almost all $x$ and all $v$

$$
\limsup _{X \rightarrow \infty} \frac{F(x, v, X)}{|X|^{p}}<\infty \text { if } p<\infty
$$

and no condition is required if $p=\infty$.

It is not difficult to show (see Lemma 2.5) that under the hypothesis (H2) the condition $\left(\mathrm{H} 3_{p}\right)$ is equivalent to the condition $\lim \sup _{X \rightarrow \infty}|F(x, v, X)| /|X|^{p}<\infty(1 \leq p<\infty)$.

Defining $F^{-}=-\inf \{F, 0\}$, we have the following result: 
Theorem 1.1 Let $\mu$ be a non-negative and finite Radon measure on $\Omega, p \in[1, \infty]$ and $F \in \mathcal{I}_{p}$. If $\left\{u_{j}\right\} \subset W_{\mu}^{1,1}\left(\Omega ; \mathbf{R}^{n}\right)$ satisfies (1.4),

$$
\left\{F^{-}\left(x, u_{j}, \nabla u_{j}\right)\right\} \text { is uniformly summable on } \Omega \text {, }
$$

and

$$
\sup _{j}\left\|\nabla u_{j}\right\|_{p ; \Omega}<\infty
$$

then

$$
\int_{\Omega} F(x, u, \nabla u) d x \leq \liminf _{j \rightarrow \infty} \int_{\Omega} F\left(x, u_{j}, \nabla u_{j}\right) d x
$$

Remark. It follows from the proof that if we replace the assumption (1.6) with

$$
\sup _{j}\left\|\frac{d D u_{j}}{d \mu}\right\|_{L_{\mu}^{p}}<\infty,
$$

then we have instead of (1.7) the conclusion

$$
\int_{\Omega} F\left(x, u, \frac{\nabla u}{a}\right) a d x \leq \liminf _{k \rightarrow \infty} \int_{\Omega} F\left(x, u_{j}, \frac{\nabla u_{j}}{a}\right) a d x,
$$

where $a=d \mu / d \mathcal{L}^{m}$.

The lower semicontinuity properties of multiple integrals with quasiconvex integrands have previously been studied in this setting by Ambrosio, Buttazzo and Fonseca in [8]. Write $\mu=a \cdot \mathcal{L}^{m}+\mu^{s}$ and $D u=\nabla u \cdot \mathcal{L}^{m}+D^{s} u$ for the Lebesgue-Radon-Nikodym decompositions with respect to Lebesgue measure and define the functional

$$
E(u)=\int_{\Omega} F\left(x, u, \frac{\nabla u}{a}\right) a d x+\int_{\Omega} G\left(x, \frac{d D^{s} u}{d \mu^{s}}\right) d \mu^{s} .
$$

Then the principal result of [8] guarantees lower semicontinuity of $E(u)$ on sequences $\left\{u_{j}\right\}$ satisfying (1.4) and (1.8), when $F$ is a Carathéodory integrand satisfying (H2) and $\left(\mathrm{H} 3_{p}\right)$, $G$ is a rank-1 convex normal integrand, $1<p<\infty$ and $a \in L^{\infty}$. It is possible to relax the conditions on $G$, see [8], and we remark that the singular part in $E(u)$ also is lower semicontinuous under the conditions of Theorem 1.1. Observe that these results easily give existence results for minimisation problems, where the discontinuity set is imposed a priori.

The proof in [8] is achieved by considering the absolutely continuous part and the singular part of $E(u)$ separately. The singular part of $E(u)$ is then treated by use of a result from [3]. In dealing with the absolutely continuous part the authors use a result from [7] on Lusin-type approximation of functions of bounded variation by Lipschitz functions. Such approximation results were first established for Sobolev functions in [37] and [1], and used in [1] to obtain lower semicontinuity results for multiple integrals on Sobolev spaces.

The main novelty of our result is that we allow $p \in[1, \infty]$ and $a=d D u / d \mathcal{L}^{m} \in L^{1}(\Omega)$. The extension from $a \in L^{\infty}$ to $a \in L^{1}$ appears to be essential for the proof of our second lower semicontinuity result stated in Theorem 1.2. Apparently this extension also requires 
a different strategy for the proof as the method based on approximation with Lipschitz functions seems to break down if $a$ is not essentially bounded. We also note that the reason that we can allow the integrand $F$ to be merely normal (and not necessarily Carathéodory) is due to our approach via Young measures.

Before stating Theorem 1.2 we recall that if $u$ is of bounded variation, then it is possible to define a measure theoretic normal $N_{u}$ to the jump set $S_{u}$ for $u$ and to define one-sided traces $u^{+}, u^{-}$of $u$ on $S_{u}$ (see Section 2). Let $D u=\nabla u \cdot \mathcal{L}^{m}+D^{s} u$ be the LebesgueRadon-Nikodym decomposition of $D u$ (with respect to Lebesgue measure). The space

$$
S B V\left(\Omega ; \mathbf{R}^{n}\right)=\left\{u \in B V\left(\Omega ; \mathbf{R}^{n}\right): D^{s} u=D^{s} u\left\lfloor S_{u}\right\}\right.
$$

of special functions of bounded variation, and its generalisation $G S B V\left(\Omega ; \mathbf{R}^{n}\right)$ (see Sections 2 and 6), were introduced by Ambrosio and De Giorgi in [10] as a natural setting for weak formulations of free discontinuity problems.

Theorem 1.2 concerns the case where $u$ is a special function of bounded variation (or, more generally, lies in $\left.G S B V\left(\Omega ; \mathbf{R}^{n}\right)\right)$ and is motivated by a compactness result due to Ambrosio (cf. [6], Theorem 2.2). The compactness result can be stated in the following manner. If $\phi$ is as in (1.2), if $\theta:[0, \infty) \rightarrow[0, \infty]$ is concave, non-decreasing and satisfies

$$
\frac{\theta(t)}{t} \rightarrow \infty \text { as } t \rightarrow 0^{+}
$$

and if $\left\{u_{j}\right\} \subset S B V\left(\Omega ; \mathbf{R}^{n}\right)$ satisfies

$$
\sup _{j}\left(\int_{\Omega}\left(\left|u_{j}\right|+\phi\left(\left|\nabla u_{j}\right|\right)\right) d x+\int_{S_{u_{j}}} \theta\left(\left|u_{j}^{+}-u_{j}^{-}\right|\right) d \mathcal{H}^{m-1}\right)<\infty,
$$

then for some subsequence of $\left\{u_{j}\right\}$ (for convenience not relabelled) and some $u \in G S B V\left(\Omega ; \mathbf{R}^{n}\right.$ )

$$
\left.\begin{array}{l}
u_{j} \rightarrow u \text { in measure, } \nabla u_{j} \rightarrow \nabla u \text { weakly in } L^{1} \\
\text { and } \sup _{j} \int_{S_{u}} \theta\left(\left|u_{j}^{+}-u_{j}^{-}\right|\right) d \mathcal{H}^{m-1}<\infty .
\end{array}\right\}
$$

For convenience we state a precise version of the compactness theorem for $G S B V$ in Section 6.

Theorem 1.2 Let $\theta$ satisfy (1.9), $p \in[1, \infty]$ and $F \in \mathcal{I}_{p}$. If $\left\{u_{j}\right\} \subset S B V\left(\Omega ; \mathbf{R}^{n}\right)$ satisfies (1.11),

$$
\left\{F^{-}\left(x, u_{j}, \nabla u_{j}\right)\right\} \text { is uniformly summable on } \Omega
$$

and

$$
\sup _{j}\left\|\nabla u_{j}\right\|_{p ; \Omega}<\infty
$$

then

$$
\int_{\Omega} F(x, u, \nabla u) d x \leq \liminf _{j \rightarrow \infty} \int_{\Omega} F\left(x, u_{j}, \nabla u_{j}\right) d x
$$


Remark. It is possible to give a more general version of Theorem 1.2 in terms of GSBV functions; we indicate how to do this in Section 6 .

Lower semicontinuity of multiple integrals with quasiconvex integrands has been studied in this setting by Ambrosio in [7] under the assumption $\theta(t) \equiv \theta_{0}>0$. The main novelty of our result is the extension to the case when $\lim _{t \rightarrow 0} \theta(t)=0$ (e.g. $\theta(t)=t^{\gamma}$, where $\gamma \in(0,1))$. We cover exactly the cases described in the compactness theorem for $G S B V$.

Consider the functional

$$
\mathcal{E}(u)=\int_{\Omega} F(x, u, \nabla u) d x+\int_{S_{u}} G\left(x, u^{+}, u^{-}, N_{u}\right) d \mathcal{H}^{m-1} .
$$

If for some constants $c_{1}, c_{2}, c_{3}, \alpha>0, \beta>1$ and $\gamma<1$ we have

$$
F(x, v, X) \geq c_{1}|v|^{\alpha}+c_{2}|X|^{\beta} \quad \text { and } \quad G(x, u, v, N) \geq \min \left\{c_{3},|u-v|^{\gamma}\right\},
$$

then by the compactness theorem the functional $\mathcal{E}$ is coercive in the space $G S B V\left(\Omega ; \mathbf{R}^{n}\right)$. Under the conditions of Theorem 1.2 the bulk energy term of $\mathcal{E}(u)$ is lower semicontinuous. Corresponding lower semicontinuity results for the surface energy in $\mathcal{E}(u)$ have been obtained by Ambrosio in [6] for the cases $\gamma \leq 0$. However, under the condition that $G$ is regularly biconvex the methods of [6] also yield lower semicontinuity of the surface energy in the case $\gamma<1$ (see also the remark following Theorem 6.2). We also notice that $\mathcal{E}(u)$ can be lower semicontinuous even though the bulk energy and surface energy are not so separately (see [19] and the references therein).

As already mentioned, we proceed as suggested by Balder in [11] and establish the lower semicontinuity results of Theorems 1.1 and 1.2 by use of Young measures. By virtue of the hypotheses of either Theorem 1.1 or Theorem 1.2 we can, by extracting a subsequence if necessary, assume that $\left\{\nabla u_{j}\right\}$ generates a Young measure $\nu=\int_{\Omega} \delta_{x} \otimes \nu_{x} d x$ (see Section 2 for notation). According to a general result from [11] (see also Theorem 2.4)

$$
\liminf _{j \rightarrow \infty} I\left(u_{j}\right) \geq \int_{\Omega} \int F(x, u(x), X) d \nu_{x}(X) d x .
$$

Since $\nabla u_{j} \rightarrow \nabla u$ weakly in $L^{1}$ it follows that almost all $\nu_{x}$ have a centre of mass (denoted by $\left.\bar{\nu}_{x}\right)$ and that $\bar{\nu}_{x}=\nabla u(x)$ almost everywhere. If, therefore, we have

$$
\int F(x, u(x), X) d \nu_{x}(X) \geq F\left(x, u(x), \bar{\nu}_{x}\right) \quad \text { a.e. }
$$

then the lower semicontinuity results of Theorems 1.1 and 1.2 follow. We express (1.16) by saying that Jensen's inequality holds for $F(x, u(x), \cdot)$ and $\nu_{x}$ for almost every $x$. The key feature of this approach is that it allows us to ignore the $(x, v)$ dependence in $F$. We establish (1.16), and hence Theorems 1.1 and 1.2, in the following manner. Let $\mathcal{Q}_{p}$ denote the class of quasiconvex functions $f: \mathbf{R}^{n \times m} \rightarrow \mathbf{R}$ satisfying the growth condition

$$
\limsup _{X \rightarrow \infty} \frac{f(X)}{|X|^{p}}<\infty
$$

if $p<\infty$; no condition is required if $p=\infty$. Note that by definition of the class $\mathcal{I}_{p}$ of admissible integrands the functions $f_{x}(X)=F(x, u(x), X)$ belong to $\mathcal{Q}_{p}$ for almost all $x$. 
The main part of the proof aims at establishing that for almost all $x$

$$
\forall f \in \mathcal{Q}_{p}: \int f d \nu_{x} \geq f\left(\bar{\nu}_{x}\right) .
$$

A result due to Kinderlehrer and Pedregal [32, 33] states that (1.18) for a fixed $x$ is equivalent to the requirement that $\left(\mathcal{L}^{m}\lfloor\Omega) \otimes \nu_{x}\right.$ be a gradient $p$-Young measure. Theorems 1.3 and 1.4 establish exactly this. Before proceeding further a few remarks are in order.

The semicontinuity result (1.15) was obtained independently by Pedregal [47] for the case of Carathéodory integrands that are bounded from below. Pedregal also emphasised the importance of Jensen's inequality (1.16) in problems of lower semicontinuity and highlighted it in his definition of closed $W^{1, p}$-quasiconvexity. This approach was also used in [31] and in [34] to obtain lower semicontinuity results for multiple integrals on $W^{1, p}$. The good localisation properties of Young measures were also used in [56] to study the principle of convergence of energies.

The key results of this paper are the following two theorems.

Theorem 1.3 Let $\mu$ be a non-negative, finite Radon measure on $\Omega$ and let $\left\{u_{j}\right\}$ be a sequence in $B V\left(\Omega ; \mathbf{R}^{n}\right)$ satisfying (1.4) and (1.6). If $\left\{\nabla u_{j}\right\}$ generates the Young measure $\nu=\int_{\Omega} \delta_{x} \otimes \nu_{x} d x$, then for $\mathcal{L}^{m}$-almost all $x,\left(\mathcal{L}^{m}\lfloor\Omega) \otimes \nu_{x}\right.$ is a gradient $p$-Young measure.

Theorem 1.4 Let $\left\{u_{j}\right\}$ be a sequence in $S B V\left(\Omega ; \mathbf{R}^{n}\right)$ satisfying (1.11) and (1.13). If $\left\{\nabla u_{j}\right\}$ generates the Young measure $\nu=\int_{\Omega} \delta_{x} \otimes \nu_{x} d x$, then for $\mathcal{L}^{m}$-almost all $x,\left(\mathcal{L}^{m}\lfloor\Omega) \otimes\right.$ $\nu_{x}$ is a gradient $p$-Young measure.

Remark. We present a more general statement of Theorem 1.4 in terms of GSBV functions in Section 6.

In some sense Theorems 1.3 and 1.4 are surprising. Quasiconvexity is defined with specific reference to gradients, but a result of Alberti [2] states that the approximate gradient $\nabla u$ of an $S B V$-function $u$ can be any summable function $V: \Omega \rightarrow \mathbf{R}^{n \times m}$. Of course, it is the conditions we impose on the measures $D u_{j}$ that force the sequence $\left\{\nabla u_{j}\right\}$ to generate a Young measure with the above property. One might think that it should be possible to decompose $\nabla u_{j}$ into a gradient and another term that converges strongly, so that the Young measure is essentially generated by the gradients. Of course, if possible, this would prove the theorems. However, by Example 7.9, this approach cannot be successful. Example 7.9 displays a sequence $\left\{u_{j}\right\}$ satisfying, simultaneously, all the conditions in Theorems 1.1-1.4, but where the sequence $\left\{\nabla u_{j}\right\}$ of approximate gradients (and any subsequence thereof) does not admit a decomposition of the form $\nabla u_{j}=E_{j}+\nabla v_{j}$, where $\left\{E_{j}\right\}$ converges strongly in $L^{1}\left(\Omega ; \mathbf{R}^{n \times m}\right)$ and $\left\{v_{j}\right\}$ weakly in $W^{1,1}\left(\Omega ; \mathbf{R}^{n}\right)$.

Theorems 1.3 and 1.4 are very close to being optimal. In Section 7 we present examples showing that if one of the conditions $(1.4),(1.6),(1.11)$ or (1.13) is slightly weakened the corresponding conclusion is false.

The proof of Theorem 1.3 is presented in Section 5. By use of a truncation argument and the Vitali-Hahn-Saks Theorem we deduce in Section 6 Theorem 1.4 from Theorem 
1.3. As noticed above Theorems 1.1 and 1.2 are then easy consequences via (1.15) and (1.16).

To prove Theorem 1.3 we exploit the characterisation of gradient Young measures due to Kinderlehrer and Pedregal [32, 33] (see also [45], [48] or [50]; [26] contains a generalisation). We only need the following special case of their result.

Lemma 1.5 (D. Kinderlehrer and P. Pedregal; special case of [32, 33]) Suppose $\nu$ is a probability measure on $\mathbf{R}^{n \times m}$ satisfying

$$
\int|X| d \nu(X)<\infty
$$

The homogeneous Young measure $\left(\mathcal{L}^{m}\lfloor\Omega) \otimes \nu\right.$ is a gradient 1 -Young measure if and only if for all quasiconvex functions $f$ for which $f(X) /|X| \rightarrow 1$ as $|X| \rightarrow \infty$ the following inequality holds:

$$
\int f(X) d \nu(X) \geq f(\bar{\nu})
$$

In the Appendix we present an almost self-contained proof of the full characterisation of gradient $p$-Young measures covering all cases $p \in[1, \infty]$. We obtain at the same time a slight refinement of the results in $[32,33]$ in the sense that we are able to show that it is only necessary to test in (1.20) with quasiconvex functions that equal $|X|$ outside large balls.

As a first step towards proving Theorem 1.3 we employ Lemma 1.5 to show that for almost all $x$ the measure $\left(\mathcal{L}^{m}\lfloor\Omega) \otimes \nu_{x}\right.$ is a gradient 1-Young measure. We establish $(1.20)$ by use of well-known results on differentiation of measures along with the following result.

Lemma 1.6 Let $f: \mathbf{R}^{n \times m} \rightarrow \mathbf{R}$ be a non-negative, quasiconvex function satisfying $f(X) /|X| \rightarrow 1$ as $X \rightarrow \infty$ and let $u: \Omega \rightarrow \mathbf{R}^{n}$ be of locally bounded variation. For $\delta \in(0,1), x \in \Omega, r \in(0, \operatorname{dist}(x, \partial \Omega)), a \in \mathbf{R}^{n}$ and $X \in \mathbf{R}^{n \times m}$ the following inequality holds:

$$
\begin{aligned}
& \int_{B_{x, r}} f(\nabla u) d y+\left|D^{s} u\right|\left(B_{x, r}\right)+\frac{1}{(1-\delta) r} \int_{B_{x, r} \backslash B_{x, \delta r}}|u(y)-(a+X(y))| d y \\
& \geq \mathcal{L}^{m}\left(B_{x, \delta r}\right) f(X) .
\end{aligned}
$$

This lemma is reminiscent of Lemma 2.5 in [9]. We derive it as a corollary of a slightly more general inequality in Section 2, the proof of which is elementary. A similar result appears to be false for quasiconvex functions with super-linear growth at infinity.

To conclude the proof of Theorem 1.3 we invoke the following technical result on truncation of sequences of gradients. More precisely the conclusion is established using Corollary 1.8 stating, in particular, that a gradient 1 -Young measure is a gradient $p$-Young measure if and only if it has a finite $p^{\text {th }}$ order moment. 
Lemma 1.7 Assume that $\Omega$ is a bounded Lipschitz domain. Let $\left\{u_{j}\right\}$ satisfy $u_{j} \rightarrow u$ weakly in $W^{1,1}(\Omega)$. Assume that for some $p \in[1, \infty]$ there is a sequence $\left\{V_{j}\right\} \subset L^{p}\left(\Omega ; \mathbf{R}^{m}\right)$ with the properties:

$$
\left\{\begin{array}{l}
\text { if } p<\infty,\left\{\left|V_{j}\right|^{p}\right\} \text { is uniformly summable on } \Omega \\
\text { if } p=\infty,\left\{V_{j}\right\} \text { is bounded in } L^{\infty}\left(\Omega ; \mathbf{R}^{m}\right)
\end{array}\right.
$$

and

$$
\nabla u_{j}-V_{j} \rightarrow 0 \text { in measure on } \Omega .
$$

Then there is $\left\{v_{j}\right\} \subset \mathcal{C}_{c}^{\infty}(\Omega)$, such that

$$
\begin{gathered}
v_{j} \rightarrow 0 \text { weakly }(\text { weakly* if } p=\infty) \text { in } W_{0}^{1, p}(\Omega), \\
\left\|\nabla u_{j}-\nabla u-\nabla v_{j}\right\|_{1 ; \Omega} \rightarrow 0
\end{gathered}
$$

and, if $p<\infty$,

$$
\left\{\left|\nabla u+\nabla v_{j}\right|^{p}\right\} \text { is uniformly summable on } \Omega \text {. }
$$

The case $p=\infty$ was treated by Zhang in [60]. An elementary proof for the cases $p<\infty$ relying on the Hodge decomposition is given in Section 3. We apply the result to vector valued functions by applying it to each coordinate function. The fact that the result is 'scalar', i.e. it is possible to prove it for real-valued functions and then transfer it to vector-valued functions by applying it to each coordinate function, paves the way for many different extensions and proofs (see [35]).

Using this result we easily derive two useful corollaries. The first concludes the proof of Theorem 1.3 and concerns the possibility of finding generating sequences for gradient Young measures with good integrability properties.

Corollary 1.8 Let $\nu=\int_{\Omega} \delta_{x} \otimes \nu_{x} d x$ be a gradient 1 -Young measure and let $u \in W^{1,1}\left(\Omega ; \mathbf{R}^{n}\right)$ be an underlying deformation. Let $p \in[1, \infty]$ and assume that $\nu$ has a finite $p^{\text {th }}$ order moment, i.e. if $p<\infty$,

$$
\int_{\Omega} \int|X|^{p} d \nu_{x}(X) d x<\infty
$$

and if $p=\infty$, there is a compact set $C \subset \mathbf{R}^{n \times m}$, such that for almost all $x$ the measure $\nu_{x}$ is carried by $C$.

Then there exists $\left\{v_{j}\right\} \subset \mathcal{C}_{c}^{\infty}\left(\Omega ; \mathbf{R}^{n}\right)$, such that $v_{j} \rightarrow 0$ weakly (weakly* if $p=\infty$ ) in $W_{0}^{1, p}\left(\Omega ; \mathbf{R}^{n}\right),\left\{\nabla u+\nabla v_{j}\right\}$ generates $\nu$ and, if $p<\infty$, the sequence $\left\{\left|\nabla u+\nabla v_{j}\right|^{p}\right\}$ is uniformly summable on $\Omega$.

Corollary 1.8 is a slight refinement of a similar result in [34], where the proof was based on a stability result from [30]. Other results in the same vein have also been obtained in [26] using arguments based on the Lusin-type approximation of general Sobolev functions with Lipschitz functions as in [1]. The first result of this kind seems to come from [33] and was obtained in an indirect way. Recently similar results have been obtained in [25] within the more general setting of compensated compactness.

The second corollary to Lemma 1.7 is the following. 
Corollary 1.9 (J. Ball and K. Zhang, [17]) Suppose $f: \mathbf{R}^{n \times m} \rightarrow \mathbf{R}$ is a quasiconvex function satisfying

$$
\limsup _{X \rightarrow \infty} \frac{f(X)}{|X|^{p}}<\infty \quad \text { if } p<\infty
$$

and no growth condition if $p=\infty$. Let $\nu=\int_{\Omega} \delta_{x} \otimes \nu_{x} d x$ be a gradient $p$-Young measure. Then

$$
\int f d \nu_{x} \geq f\left(\bar{\nu}_{x}\right)
$$

holds for almost all $x \in \Omega$.

The proof in [17] is based on the lower semicontinuity result in [1] and Chacon's Biting Lemma.

By virtue of (1.15) it is clear that Theorems 1.1 and 1.2 follow from Theorems 1.3, 1.4 and Corollary 1.9.

It is possible to prove Theorems 1.1 and 1.2 in the case $p \in(1, \infty)$ without relying on the characterisation of gradient Young measures. The proof then relies on Lemma 1.6 and the following approximation result, which again is proved using Lemma 1.6, Corollaries 1.8 and 1.9 .

Proposition 1.10 Let $f: \mathbf{R}^{n \times m} \rightarrow \mathbf{R}$ be a quasiconvex function, such that for some $c_{1}$, $c_{2}>0$ and $p>1$

$$
c_{1}|X|^{p}-c_{2} \leq f(X) \leq c_{2}\left(|X|^{p}+1\right)
$$

holds for all $X$. Then there exist $f_{j}: \mathbf{R}^{n \times m} \rightarrow \mathbf{R}$ that are quasiconvex and satisfy

(a) $f_{j}(X) \leq f_{j+1}(X)$,

(b) $f_{j}(X) \rightarrow f(X)$ as $j \rightarrow \infty$,

(c) there exist $a_{j}, r_{j}>0, b_{j} \in \mathbf{R}$, such that

$$
f_{j}(X)=f_{j}^{\star \star}(X)=a_{j}|X|+b_{j} \text { if }|X| \geq r_{j},
$$

where $f_{j}^{\star \star}$ denotes the convex envelope of $f_{j}$.

The approximation result in [40] implies the existence of quasiconvex functions $g_{j}$ satisfying (a), (b) and

$\left(c^{\prime}\right)$ there exist $r_{j}>0$, such that $g_{j}(X)=c_{1}|X|^{p}-c_{2}$ if $|X| \geq r_{j}$.

The proof in [40] is based on a result on higher integrability of certain minimising sequences. For our purposes it is important that we have (c) and not (c').

We note that assumption (1.24) cannot be avoided. In Example 7.4 we show by use of results from [15] and [54] that the polyconvex function $f(X)=|X|^{p}+|\operatorname{det} X|$, where $p \in[1,2)$, defined on $\mathbf{R}^{2 \times 2}$, cannot be approximated from below by sub-quadratic rank-1 convex functions. 
The paper is organised as follows. In Section 2 we briefly recall the main definitions and state some preliminary results. Section 3 is devoted to proving the Decomposition Lemma (Lemma 1.7) and its corollaries. In Section 4 we give the proof of Proposition 1.10. The main results, Theorems 1.1-1.4, are proved in Sections 5 and 6. In Section 7 we have gathered some examples that illustrate the sharpness of our hypotheses. Section 8 is an appendix and contains an essentially self-contained proof of the characterisation of gradient Young measures.

The present paper is a revised and extended version of an earlier manuscript, where the most important changes are that Proposition 1.10 and the full proof of the characterisation of gradient Young measures have been included. The result stated in Theorems 1.1 and 1.3 together with a proof based on [9] was announced at the workshop 'Calculus of Variations and Nonlinear Elasticity' in Cortona, Italy, June 1995.

\section{Notation and preliminary results}

In this section we gather some definitions and elementary results that are used in the sequel.

\subsection{Basic notation}

Our main references for measure theory are [23] and [51]. Except for the Hausdorff measure $\mathcal{H}^{m-1}$ all measures occurring in this paper are Radon measures. If $\mu$ is a measure and $A$ is a set, then the measure $\mu\lfloor A$ is defined as $(\mu\lfloor A)(B)=\mu(A \cap B)$.

Let $O$ be either an open or a compact subset of $\mathbf{R}^{D}$ and let $\mathbf{B}(O)$ denote the $\sigma$-field of Borel subsets. For a bounded $\mathbf{R}^{d}$-valued Radon measure on $O$ the total variation on $A \in \mathbf{B}(O)$ is

$$
|\lambda|(A)=\sup \left\{\sum_{i=1}^{\infty}\left|\lambda\left(A_{i}\right)\right|\right\},
$$

where the supremum is taken over all partitions of $A$ into countably many Borel subsets $A_{i}$ and $\left|\lambda\left(A_{i}\right)\right|$ denotes the usual Euclidean norm of $\lambda\left(A_{i}\right)$. The function $|\lambda|$ is called the total variation measure for $\lambda$ and is a non-negative, finite Radon measure on $O$.

As the concepts of uniform absolute continuity and uniform summability are central to the present work we display a formal definition.

Definition Let $\mu$ be a non-negative, finite Radon measure on $O$. A family $\Lambda$ of $\mathbf{R}^{d}$-valued bounded Radon measures on $O$ is said to be uniformly absolutely continuous with respect to $\mu$, briefly uniformly $\mu-A C$, if for any $\varepsilon>0$ there exists a $\delta>0$, such that for $B \in \mathbf{B}(O)$

$$
\mu(B)<\delta \Rightarrow|\lambda(B)|<\varepsilon \text { for all } \lambda \in \Lambda .
$$

$A$ family $F$ of $\mu$ summable functions $V: O \rightarrow \mathbf{R}^{d}$ is uniformly $\mu$ summable if the family $\{V \cdot \mu: V \in F\}$ is uniformly $\mu-A C$. 
Remarks. 1. By the Radon-Nikodym Theorem $\Lambda$ is uniformly $\mu$-AC if and only if each measure in $\Lambda$ is absolutely continuous with respect to $\mu$ and the family $F=\{d \lambda / d \mu: \lambda \in$ $\Lambda$ \} of Radon-Nikodym derivatives is uniformly $\mu$ summable.

2. If $\mu$ has no atoms and $\left\{\lambda_{j}\right\}$ is uniformly $\mu$-AC, then $\sup _{j}\left|\lambda_{j}\right|(O)<\infty$.

3. $\left\{\lambda_{j}\right\}$ is uniformly $\mu$ - AC if and only if the sequence $\left\{\left|\lambda_{j}\right|\right\}$ of total variation measures is uniformly $\mu$-AC.

Denote by $\mathcal{C}^{k}\left(O ; \mathbf{R}^{d}\right)$ the space of $\mathbf{R}^{d}$-valued $C^{k}$-functions on $O$. The subspace of functions with a compact support is denoted by $\mathcal{C}_{c}^{k}\left(O ; \mathbf{R}^{d}\right)$. If $d=1$ we simply write $\mathcal{C}^{k}(O)$ instead; similarly for all other function spaces.

Denote by $\mathcal{C}_{0}^{0}\left(O ; \mathbf{R}^{d}\right)$ the space of $\mathbf{R}^{d}$-valued continuous functions $\varphi$ with the property: for every $\varepsilon>0$ there is a compact set $K \subseteq O$, such that $|\varphi(x)| \leq \varepsilon$ if $x \in O \backslash K$. Of course, if $O$ is compact, $\mathcal{C}^{0}\left(O ; \mathbf{R}^{d}\right)=\mathcal{C}_{0}^{0}\left(O ; \mathbf{R}^{d}\right)$. Endowed with the supremum norm, denoted by $\|\cdot\|_{\infty ; O}, \mathcal{C}_{0}^{0}\left(O ; \mathbf{R}^{d}\right)$ is a separable Banach space. By the Riesz Theorem the dual space $\mathcal{C}_{0}^{0}\left(O ; \mathbf{R}^{d}\right)^{\prime}$ can by the duality pairing

$$
\langle\lambda ; \varphi\rangle=\int_{O} \varphi(x) \cdot \frac{d \lambda}{d|\lambda|}(x) d|\lambda|(x)
$$

be identified with the space of bounded $\mathbf{R}^{d}$-valued Radon measures on $O$. The corresponding norm of $\lambda$ is $\|\lambda\|=|\lambda|(O)$.

Our reference for approximate limits and derivatives is [6]. Let $O$ be an open subset of $\mathbf{R}^{D}$ and $u: O \rightarrow \mathbf{R}^{d}$ a Borel function. We take $S=\mathbf{R}^{d} \cup\{\infty\}$ to be the one point compactification of $\mathbf{R}^{d}$ and consider $u$ as a function with values in $S$. Let $d$ be a compatible metric on $S$. Take $F \in \mathbf{B}(O)$ and $x_{0} \in O$ with the property $\mathcal{L}^{D}\left(F \cap B_{x_{0}, r}\right)>0$ for all $r>0$. Here and throughout the paper $B_{x_{0}, r}$ denotes the open ball centred at $x_{0}$ with radius $r$.

Approximate limit: $v \in S$ is said to be an approximate limit in $x_{0}$ for $u$ in the domain $F$, written

$$
v=\operatorname{ap} \lim _{\substack{x \rightarrow x_{0} \\ x \in F}} u(x)
$$

if

$$
\lim _{r \rightarrow 0^{+}} f_{B_{x_{0}, r} \cap F} d(u(x), v) d x=0 .
$$

The approximate limit is unique if it exists.

Jump set: The jump set $S_{u}$ of $u$ is defined as the set of points where $u$ has no approximate limit, i.e.

$$
S_{u}=\left\{x \in O: \operatorname{ap} \lim _{\substack{y \rightarrow x \\ y \in O}} u(y) \text { does not exist }\right\} .
$$

The set $S_{u}$ is Borel, $\mathcal{L}^{D}\left(S_{u}\right)=0$ and

$$
u(x)=\operatorname{ap} \lim _{\substack{y \rightarrow x \\ y \in O}} u(y)
$$

for almost all $x \in O \backslash S_{u}$. In case (2.1) holds at $x$ we say that $u$ is approximately continuous at $x$. 
Approximate gradients: The function $u: O \rightarrow \mathbf{R}^{d}$ is approximately differentiable at $x_{0} \in \Omega$ if it is approximately continuous at $x_{0}$ and if there exists a matrix $X \in \mathbf{R}^{d \times D}$, such that

$$
\operatorname{ap} \lim _{\substack{x \rightarrow x_{0} \\ x \in O}} \frac{\left|u(x)-u\left(x_{0}\right)-X\left(x-x_{0}\right)\right|}{\left|x-x_{0}\right|}=0 .
$$

In case $u$ is approximately differentiable at $x_{0}$ the matrix $X$ in (2.2) is uniquely determined and is called the approximate gradient of $u$ at $x_{0}$. It is denoted by $\nabla u\left(x_{0}\right)$. The set of points $\nabla_{u}$, where $u$ is approximately differentiable is a Borel set and the function $\nabla u: \nabla_{u} \rightarrow \mathbf{R}^{d \times D}$ is a Borel function.

Our references for function spaces are [61] and [6] for $B V$ and $S B V$. We use the notations

$$
\|u\|_{p ; O}=\|u\|_{L^{p}\left(O ; \mathbf{R}^{d}\right)},\|u\|_{1, p ; O}=\|u\|_{W^{1, p}\left(O ; \mathbf{R}^{d}\right)}
$$

and if $O=\mathbf{R}^{D}$ we omit $O$ from the notation. The distributional gradient of a distribution $u$ is denoted by $D u$. In particular, if $u: O \rightarrow \mathbf{R}^{d}$ is of bounded variation, then $D u=$ $\left\{\partial u_{r} / \partial x_{s}\right\}$ is a $\mathbf{R}^{d \times D}$-valued bounded Radon measure on $O$. Furthermore, in this case $u$ is approximately differentiable almost everywhere and

$$
\frac{1}{r} f_{B_{x, r}}|u(y)-u(x)-\nabla u(x)(y-x)| d y \rightarrow 0 \text { as } r \rightarrow 0^{+}
$$

$\mathcal{L}^{D}$ almost everywhere. The Lebesgue-Radon-Nikodym decomposition of $D u$ takes the form

$$
D u=\nabla u \cdot \mathcal{L}^{D}+D^{s} u,
$$

where $D^{s} u$ is a singular measure. The jump set $S_{u}$ of $u$ is countably $(D-1)$-rectifiable, i.e.

$$
S_{u}=\bigcup_{i=1}^{\infty} K_{i} \cup N
$$

where $\mathcal{H}^{D-1}(N)=0$ and $K_{i}$ are compact sets, each contained in an embedded $\mathcal{C}^{1}$ hypersurface , ${ }_{i}$. There exists a Borel function $N_{u}: S_{u} \rightarrow \mathbf{R}^{D}$, such that $\left|N_{u}(x)\right|=1$ for all $x$ and such that $N_{u}(x)$ is normal $\mathcal{H}^{D-1}$-almost everywhere in $K_{i}$ to the surface, ${ }_{i}$. We refer to $N_{u}$ as a unit normal to $S_{u}$; it is clearly not unique.

If corresponding to a unit vector $N$ and a point $x$ we define the half-spaces

$$
\begin{aligned}
& \pi^{+}(x, N)=\left\{y \in \mathbf{R}^{D}:(y-x) \cdot N>0\right\}, \\
& \pi^{-}(x, N)=\left\{y \in \mathbf{R}^{D}:(y-x) \cdot N<0\right\},
\end{aligned}
$$

then the approximate limits

$$
u^{+}\left(x, N_{u}(x)\right)=\text { ap } \lim _{\substack{y \rightarrow x \\ y \in \pi^{+}\left(x, N_{u}(x)\right)}} u(y), \quad u^{-}\left(x, N_{u}(x)\right)=\text { ap } \lim _{\left.y \in \pi^{-} \rightarrow x, N_{u}(x)\right)} u(y)
$$

exist for $\mathcal{H}^{D-1}$-almost all $x \in S_{u}$. We suppress the dependence on $N_{u}$ and write simply $u^{+}=u^{+}(x)$ and $u^{-}=u^{-}(x)$. Notice that the matrix $\left(u^{+}-u^{-}\right) \otimes N_{u}$ is uniquely determined 
$\mathcal{H}^{D-1}$-almost everywhere on $S_{u}$. The Lebesgue decomposition of $D^{s} u$ with respect to $D^{s} u\left\lfloor S_{u}\right.$ is

$$
D^{s} u=C u+\left(u^{+}-u^{-}\right) \otimes N_{u} \cdot \mathcal{H}^{D-1}\left\lfloor S_{u},\right.
$$

where $C u$ is a singular measure, such that $|C u|(A)=0$ when $A \in \mathbf{B}(O)$ and $\mathcal{H}^{D-1}(A)<$ $\infty$. A function $u: O \rightarrow \mathbf{R}^{d}$ is a special function of bounded variation, briefly $u \in$ $S B V\left(O ; \mathbf{R}^{d}\right)$, if $u$ is of bounded variation and $C u=0$, or equivalently, $D^{s} u=D^{s} u\left\lfloor S_{u}\right.$.

\subsection{Integrands and Young measures}

Definition (L.C. Young, [59].) A Young measure on $\Omega \times \mathbf{R}^{d}$ is a non-negative Radon measure $\nu$ on $\Omega \times \mathbf{R}^{d}$ with the property $\nu\left(A \times \mathbf{R}^{d}\right)=\mathcal{L}^{m}(A)$ for all Borel subsets $A$ of $\Omega$.

Remark. The definition of Young measure used here follows that of Berliocchi and Lasry [18]. It can be shown to be equivalent to the original definition due to Young and the ones used in e.g. [11], [13], [45], [48] and [50].

Notice that a product measure on $\Omega \times \mathbf{R}^{d}$ of the form $\left(\mathcal{L}^{m}\lfloor\Omega) \otimes \nu^{\prime}\right.$ is a Young measure on $\Omega \times \mathbf{R}^{d}$ exactly if $\nu^{\prime}$ is a probability measure on $\mathbf{R}^{d}$. Such Young measures are called homogeneous. Often it is clear from the context that all Young measures considered are Young measures on some specific set and in such cases we simply speak of Young measures.

Definition An elementary Young measure is a Young measure $\nu$ for which there exists $a \mathcal{L}^{m}$ measurable mapping $V: \Omega \rightarrow \mathbf{R}^{d}$, such that

$$
\int_{\Omega \times \mathbf{R}^{d}} f d \nu=\int_{\Omega} f(x, V(x)) d x
$$

for all $f \in \mathcal{C}_{0}^{0}\left(\Omega \times \mathbf{R}^{d}\right)$.

Remark. If $\nu$ is an elementary Young measure as above, then we write

$$
\nu=\varepsilon_{V}=\int_{\Omega} \delta_{x} \otimes \delta_{V(x)} d x
$$

where $\delta_{x}$ is the Dirac measure on $\Omega$ concentrated at $x$ and $\delta_{V(x)}$ is the Dirac measure on $\mathbf{R}^{d}$ concentrated at $V(x)$.

Proposition 2.1 ([20], Proposition 13 pp. 39-40.) Let $\nu$ be a Young measure on $\Omega \times \mathbf{R}^{d}$. Then there exists a mapping $x \mapsto \nu_{x}$ from $\Omega$ into $\mathcal{C}_{0}^{0}\left(\mathbf{R}^{d}\right)^{\prime} \cap\{\lambda: \lambda \geq 0\}$, the set of non-negative, finite Radon measures on $\mathbf{R}^{d}$ with the following properties.

(i) For any Borel function $f: \Omega \times \mathbf{R}^{d} \rightarrow[0, \infty]$ the function $x \mapsto \int_{\mathbf{R}^{d}} f(x, X) d \nu_{x}(X)$ is $\mathcal{L}^{m}$ measurable and

$$
\int_{\Omega \times \mathbf{R}^{d}} f d \nu=\int_{\Omega} \int_{\mathbf{R}^{d}} f(x, X) d \nu_{x}(X) d x
$$

(ii) $\nu_{x}\left(\mathbf{R}^{d}\right)=1$ for $\mathcal{L}^{m}$-almost all $x$.

Furthermore, if $x \mapsto \nu_{x}^{\prime}$ is another such mapping, then $\nu_{x}^{\prime}=\nu_{x}$ for $\mathcal{L}^{m}$-almost all $x$. 
Remarks. 1. Our Proposition 2.1 is a special case of Proposition 13 in [20].

2. We summarise the content of (i) by writing $\nu=\int_{\Omega} \delta_{x} \otimes \nu_{x} d x$.

Let $\left\{V_{j}\right\}$ be a sequence of measurable mappings of $\Omega$ into $\mathbf{R}^{d}$. Since the corresponding sequence $\left\{\varepsilon_{V_{j}}\right\}$ of elementary Young measures is always bounded in $\mathcal{C}_{0}^{0}\left(\Omega \times \mathbf{R}^{d}\right)^{\prime}$ it follows from Alaouglu's Compactness Theorem that there is a subsequence $\left\{V_{j_{k}}\right\}$ and a measure $\nu \in \mathcal{C}_{0}^{0}\left(\Omega \times \mathbf{R}^{d}\right)^{\prime}$, such that

$$
\varepsilon_{V_{j_{k}}} \rightarrow \nu \text { weakly* in } \mathcal{C}_{0}^{0}\left(\Omega \times \mathbf{R}^{d}\right)^{\prime}
$$

The following lemma characterises the case where $\nu$ is a Young measure.

Lemma 2.2 (N. Hungerbühler [29], Kristensen [34].) Under the above assumptions the measure $\nu$ is a Young measure if and only if

$$
\sup _{k} \mathcal{L}^{m}\left(\left\{x \in \Omega:\left|V_{j_{k}}(x)\right| \geq t\right\}\right) \rightarrow 0 \text { as } t \rightarrow \infty
$$

The condition (2.4) is equivalent to the following condition: there exists a Borel function $h: \mathbf{R}^{d} \rightarrow[0, \infty]$, such that $h(X) \rightarrow \infty$ as $X \rightarrow \infty$ and

$$
\sup _{k} \int_{\Omega} h\left(V_{j_{k}}\right) d x<\infty
$$

Remark. In case (2.3) and (2.4) hold we say that the sequence $\left\{V_{j_{k}}\right\}$ generates the Young measure $\nu$.

The next lemma is well-known and is easily proved using Lemma 2.2.

Lemma 2.3 Let $\left\{V_{j}\right\},\left\{W_{j}\right\}$ be two sequences of measurable mappings of $\Omega$ into $\mathbf{R}^{d}$. If $\left\{V_{j}\right\}$ generates the Young measure $\nu$ and if $V_{j}-W_{j} \rightarrow 0$ in measure, then also $\left\{W_{j}\right\}$ generates the Young measure $\nu$.

Definition An extended real-valued function $F: \Omega \times \mathbf{R}^{d} \rightarrow \mathbf{R} \cup\{ \pm \infty\}$ is called a normal integrand if $F(x, v)>-\infty$ everywhere, if $F$ is Borel measurable and if for every fixed $x \in \Omega$ the partial function $F(x, \cdot): \mathbf{R}^{d} \rightarrow \mathbf{R} \cup\{ \pm \infty\}$ is lower semicontinuous.

Definition A real-valued function $F: \Omega \times \mathbf{R}^{d} \rightarrow \mathbf{R}$ is called a Carathéodory integrand if both $F$ and $-F$ are normal integrands.

In the statement of the next theorem we use the notation $F^{-}=-\inf \{F, 0\}$.

Theorem 2.4 Let $\left\{V_{j}\right\}$ be a sequence of measurable mappings of $\Omega$ into $\mathbf{R}^{d}$ and assume that it generates the Young measure $\nu$. If $F: \Omega \times \mathbf{R}^{d} \rightarrow \mathbf{R} \cup\{ \pm \infty\}$ is a normal integrand and if $\left\{F^{-}\left(\cdot, V_{j}\right)\right\}$ is uniformly summable, then

$$
\liminf _{j \rightarrow \infty} \int_{\Omega} F\left(x, V_{j}(x)\right) d x \geq \int F d \nu
$$


If additionally $F$ is a Carathéodory integrand, then $\left\{F\left(\cdot, V_{j}\right)\right\}$ is uniformly summable on $\Omega$ if and only if

$$
\lim _{j \rightarrow \infty} \int_{\Omega} F\left(x, V_{j}(x)\right) d x=\int F d \nu
$$

Proof. The proof can be obtained by mimicking the proof of Theorem 2.6 in [5]. The first part of the theorem and the 'if' part of the second assertion are proved in [11] and in [18]. The 'only if' part of the second assertion of the theorem is proved in [33].

Definition (D. Kinderlehrer and P. Pedregal, [32,33].) Let $p \in[1, \infty]$. A Young measure $\nu$ on $\Omega \times \mathbf{R}^{n \times m}$ is a gradient $p$-Young measure if there exists a sequence $\left\{u_{j}\right\}$ in $W^{1, p}\left(\Omega ; \mathbf{R}^{n}\right)$, such that

(i) $\quad\left\{u_{j}\right\}$ is weakly (weakly $*^{*}$ if $p=\infty$ ) convergent in $W^{1, p}\left(\Omega ; \mathbf{R}^{n}\right)$;

(ii) $\varepsilon_{\nabla u_{j}} \rightarrow \nu$ weakly* in $\mathcal{C}_{0}^{0}\left(\Omega \times \mathbf{R}^{n \times m}\right)^{\prime}$.

Remark. We call the limit $u$ of $\left\{u_{j}\right\}$ an underlying deformation for $\nu$. It follows from Theorem 2.4 that if $\nu=\int_{\Omega} \delta_{x} \otimes \nu_{x} d x$, then for almost all $x$ the probability measure $\nu_{x}$ has a centre of mass $\bar{\nu}_{x}$ and

$$
\bar{\nu}_{x}=\nabla u(x)
$$

We end this subsection with an elementary, but very useful observation. Suppose that $\left\{V_{j}\right\}$ generates the Young measure $\nu=\int_{\Omega} \delta_{x} \otimes \nu_{x} d x$ and that $V: \Omega \rightarrow \mathbf{R}^{d}$ is a measurable mapping. Then the sequence $\left\{V_{j}+V\right\}$ generates a Young measure

$$
\lambda=\int_{\Omega} \delta_{x} \otimes\left(\nu_{x} \star \delta_{V(x)}\right) d x,
$$

where $\nu_{x} \star \delta_{V(x)}$ denotes the convolution of the two measures $\nu_{x}$ and $\delta_{V(x)}$ defined as

$$
\left\langle\nu_{x} \star \delta_{V(x)} ; f\right\rangle=\int_{\mathbf{R}^{d}} f(v+V(x)) d \nu_{x}(v), f \in \mathcal{C}_{0}^{0}\left(\mathbf{R}^{d}\right) .
$$

Observe that convolution with $\delta_{V(x)}$ simply corresponds to a translation with $V(x)$.

\subsection{Quasiconvexity}

Definition (C.B. Morrey, [43].) A function $f: \mathbf{R}^{n \times m} \rightarrow \mathbf{R} \cup\{ \pm \infty\}$ is quasiconvex at $X \in \mathbf{R}^{n \times m}$ if for every open and bounded set $\omega \subset \mathbf{R}^{m}$ with $\mathcal{L}^{m}(\partial \omega)=0$ one has

$$
\int_{\omega} f(X+\nabla u(x)) d x \geq \int_{\omega} f(X) d x=\mathcal{L}^{m}(\omega) f(X)
$$

for all $u \in W_{0}^{1, \infty}\left(\omega ; \mathbf{R}^{n}\right)$ for which the integral on the left hand side exists. The function $f$ is quasiconvex if it is quasiconvex at every $X \in \mathbf{R}^{n \times m}$.

Remarks. 1. It is enough to know that (2.8) holds for one (non-empty) open and bounded set $\omega$. Indeed, if (2.8) holds for one open and bounded set $\omega=\Omega$ (not necessarily with 
$\left.\mathcal{L}^{m}(\partial \Omega)=0\right)$, then it holds for all open and bounded sets $\omega$ with $\mathcal{L}^{m}(\partial \omega)=0$. If $f<\infty$, then the condition that $\mathcal{L}^{m}(\partial \omega)=0$ can be omitted (see e.g. [45]).

2. It can be shown (see e.g. [45]) that a real-valued quasiconvex function is rank-1 convex, i.e. $f$ is convex on rank-1 lines in $\mathbf{R}^{n \times m}$.

A function $f: \mathbf{R}^{n \times m} \rightarrow \mathbf{R} \cup\{ \pm \infty\}$ is separately convex if it is convex on lines parallel to the coordinate axes. It can be shown that a real-valued separately convex function is locally Lipschitz continuous (see [21]). Furthermore we have the following elementary lemma.

Lemma 2.5 Suppose that $f: \mathbf{R}^{n \times m} \rightarrow \mathbf{R}$ is separately convex and that for some $p \in$ $[1, \infty)$

$$
\limsup _{X \rightarrow \infty} \frac{f(X)}{|X|^{p}}<\infty
$$

Then also

$$
\limsup _{X \rightarrow \infty} \frac{|f(X)|}{|X|^{p}}<\infty
$$

Proof. For $R>0$ the following inequality holds:

$$
2^{m n} f(0)-\inf _{|X| \leq R} f(X) \leq\left(2^{m n}-1\right) \sup _{|X| \leq R} f(X)
$$

The lemma follows from this. To derive (2.9) we take $X^{1}$, such that $\left|X^{1}\right| \leq R$ and $f\left(X^{1}\right)=\inf _{|X| \leq R} f(X)$. Let $X^{1}, X^{2}, \ldots, X^{2^{m n}}$ denote the orbit of $X^{1}$ under reflections in the coordinate hyperplanes (it is not assumed that the $X^{i}$ 's are distinct). By separate convexity

$$
2^{m n} f(0) \leq \sum_{i=1}^{2^{m n}} f\left(X^{i}\right),
$$

and since $\left|X^{i}\right| \leq R$ inequality (2.9) follows.

Definition Let $f: \mathbf{R}^{n \times m} \rightarrow \mathbf{R} \cup\{ \pm \infty\}$ be an extended real-valued function. The quasiconvex envelope $f^{q c}$ of $f$ is defined as

$$
f^{q c}(X)=\sup \{g(X): g \text { quasiconvex and } g \leq f\} .
$$

Remark. It is not excluded that $f^{q c} \equiv-\infty$.

Lemma 2.6 (D. Kinderlehrer and P. Pedregal, [32] the appendix.) Let $f: \mathbf{R}^{n \times m} \rightarrow \mathbf{R}$ be a continuous function. Let an open and bounded set $\omega \subset \mathbf{R}^{m}$ with $\mathcal{L}^{m}(\partial \omega)=0$ be given and define the extended real-valued function $Q_{\omega} f: \mathbf{R}^{n \times m} \rightarrow \mathbf{R} \cup\{ \pm \infty\}$ as

$$
Q_{\omega} f(X)=\inf \left\{f_{\omega} f(X+\nabla u(x)) d x: u \in W_{0}^{1, \infty}\left(\omega ; \mathbf{R}^{n}\right)\right\} .
$$

Then $Q_{\omega} f=f^{q c}$. 
Lemma 2.7 (B. Yan, [58] Lemma 3.1.) Let $f: \mathbf{R}^{n \times m} \rightarrow \mathbf{R}$ be a continuous function and let $B=\left\{x \in \mathbf{R}^{n}:|x|<1\right\}$. For any $X \in \mathbf{R}^{n \times m}$ there is a sequence $\left\{u_{j}\right\}$ in $W_{0}^{1, \infty}\left(B ; \mathbf{R}^{n}\right)$, such that

$$
f_{B} f\left(X+\nabla u_{j}\right) d x \rightarrow f^{q c}(X) \text { and }\left\|u_{j}\right\|_{\infty ; B} \rightarrow 0
$$

For quasiconvex functions of linear growth at infinity we have the following elementary lemma, which seems to have been overlooked in the literature.

Lemma 2.8 Let $f: \mathbf{R}^{n \times m} \rightarrow \mathbf{R}$ be a quasiconvex function satisfying

$$
\limsup _{X \rightarrow \infty} \frac{|f(X)|}{|X|}<\infty
$$

and define $f^{\infty}(X)=\lim \sup _{t \rightarrow \infty} f(t X) / t$. Let $\Omega$ be a bounded Lipschitz domain in $\mathbf{R}^{m}$ and denote by $N_{\partial \Omega}$ the outward unit normal on $\partial \Omega$. For $u \in W^{1,1}\left(\Omega ; \mathbf{R}^{n}\right), a \in \mathbf{R}^{n}$ and $X \in \mathbf{R}^{n \times m}$ the inequality

$$
\mathcal{L}^{m}(\Omega) f(X) \leq \int_{\Omega} f(\nabla u) d x+\int_{\partial \Omega} f^{\infty}\left((a+X x-u(x)) \otimes N_{\partial \Omega}(x)\right) d \mathcal{H}^{m-1}(x)
$$

holds.

Remarks. 1. The recession function $f^{\infty}$ is a positively 1-homogeneous, Lipschitz continuous function.

2. By the Divergence Theorem $\int_{\Omega} \nabla u d x=\int_{\partial \Omega} u \otimes N_{\partial \Omega} d \mathcal{H}^{m-1}$.

Proof. It suffices to prove the assertion for $a=0$ and $X=0$. Since $\Omega$ is a Lipschitz domain we can assume that $u \in W^{1,1}\left(\mathbf{R}^{m} ; \mathbf{R}^{n}\right)$. Let $\left\{\varphi_{t}\right\}_{t>0}$ be a standard $\mathcal{C}^{\infty}$-mollifier and define for $\varepsilon, \delta \in(0,1)$ the convolutions

$$
\eta_{\varepsilon}=\varphi_{\varepsilon} \star 1_{\Omega} \quad \text { and } \quad u_{\delta}=\varphi_{\delta} \star u
$$

Observe that $\eta_{\varepsilon} \varphi_{\delta} \equiv 0$ outside $\Omega_{\varepsilon}=\{x: \operatorname{dist}(x, \Omega)<\varepsilon\}$ and consequently, since $f$ is quasiconvex at 0 ,

$$
\mathcal{L}^{m}\left(\Omega_{\varepsilon}\right) f(0) \leq \int_{\Omega_{\varepsilon}} f\left(\eta_{\varepsilon} \nabla u_{\delta}+u_{\delta} \otimes \nabla \eta_{\varepsilon}\right) d x
$$

We now employ an auxiliary function as in [9]. For each $x \in \Omega_{\varepsilon}$ we introduce the positively 1-homogeneous, Lipschitz function

$$
g_{x}(X)=\sup _{t>0} \frac{f\left(\eta_{\varepsilon}(x) \nabla u_{\delta}(x)+t X\right)-f\left(\eta_{\varepsilon}(x) \nabla u_{\delta}(x)\right)}{t} .
$$

By rank-1 convexity of $f, g_{x}(X)=f^{\infty}(X)$ whenever $\operatorname{rank} X \leq 1$, and therefore

$$
f\left(\eta_{\varepsilon} \nabla u_{\delta}+u_{\delta} \otimes \nabla \eta_{\varepsilon}\right) \leq f\left(\eta_{\varepsilon} \nabla u_{\delta}\right)+f^{\infty}\left(u_{\delta} \otimes \nabla \eta_{\varepsilon}\right) .
$$


Consequently,

$$
\mathcal{L}^{m}\left(\Omega_{\varepsilon}\right) f(0) \leq \int_{\Omega_{\varepsilon}} f\left(\eta_{\varepsilon} \nabla u_{\delta}\right) d x+\int_{\Omega_{\varepsilon}} f^{\infty}\left(u_{\delta} \otimes \nabla \eta_{\varepsilon}\right) d x
$$

and therefore letting $\varepsilon \rightarrow 0^{+}$we obtain by virtue of Reshetnyak's Continuity Theorem (see [49] Theorem 3)

$$
\mathcal{L}^{m}(\Omega) f(0) \leq \int_{\Omega} f\left(\nabla u_{\delta}\right) d x+\int_{\partial \Omega} f^{\infty}\left(-u_{\delta} \otimes N_{\partial \Omega}\right) d \mathcal{H}^{m-1}
$$

Finally we conclude by letting $\delta \rightarrow 0^{+}$and applying e.g. Theorem 5.10 .7 of [61].

Proof of Lemma 1.6. Let $\left\{\varphi_{\varepsilon}\right\}_{\varepsilon>0}$ be a standard $\mathcal{C}^{\infty}$-mollifier and put $u_{\varepsilon}=\varphi_{\varepsilon} \star u$. Note that if $\varepsilon>0$ is sufficiently small, then $u_{\varepsilon}$ is well-defined and smooth on $B_{x, r}$. Because the function $t \mapsto \int_{\partial B_{x, t}}\left|u_{\varepsilon}(y)-(a+X y)\right| d \mathcal{H}^{m-1}(y)$ is continuous on $[\delta r, r]$ we can find $R \in[\delta r, r]$, such that

$$
\int_{\partial B_{x, R}}\left|u_{\varepsilon}(y)-(a+X y)\right| d \mathcal{H}^{m-1}(y) \leq \frac{1}{r(1-\delta)} \int_{B_{x, r} \backslash B_{x, \delta r}}\left|u_{\varepsilon}(y)-(a+X y)\right| d y .
$$

If we apply (2.10) with $\Omega=B_{x, R}$, notice that $f^{\infty}(X)=|X|$ and make appropriate use of $f \geq 0$ we get (1.21) with $u_{\varepsilon}$ in place of $u$. We conclude the proof by letting $\varepsilon$ tend to 0 and using Reshetnyak's Continuity Theorem (see [49], Theorem 3).

It is also possible to prove (1.21) directly by means of an argument, which is similar to the proof of Lemma 2.5 in [9].

\section{Proof of the Decomposition Lemma}

In this section we prove Lemma 1.7 and its corollaries. The proof of Lemma 1.7 in the case $p<\infty$ is obtained in three steps each stated as a lemma. The main tool for the proof is the Hodge decomposition, which is used in the last step. The case $p=\infty$ is as noted in the Introduction a result due to Zhang.

Lemma 3.1 Suppose that $\Omega$ is a bounded Lipschitz domain. Let $p \in[1, \infty)$, let $\left\{u_{j}\right\}$ be a sequence, which converges weakly to 0 in $W^{1, p}(\Omega)$ and for which $\left\{\left|D u_{j}\right|^{p}\right\}$ is uniformly summable on $\Omega$. Then there exists a sequence $\left\{v_{j}\right\}$ in $\mathcal{C}_{c}^{\infty}(\Omega)$, such that

$$
u_{j}-v_{j} \rightarrow 0 \text { strongly in } W^{1, p}(\Omega)
$$

Proof. To simplify notation we assume that $\Omega=\{x:|x|<1\}$. The proof in the general case is analogous. By virtue of the Rellich-Kondrachov Compactness Theorem

$$
\delta_{j}=\sqrt{\left\|u_{j}\right\|_{p ; \Omega}} \rightarrow 0 .
$$


Take a cut-off functions $\eta_{j}: \Omega \rightarrow[0,1]$ compactly supported in $\Omega$ and with $\eta_{j}=1$ on $B_{0, \delta_{j}}$ and $\operatorname{Lip}\left(\eta_{j}\right) \leq 2 / \delta_{j}$. Define $w_{j}=\eta_{j} u_{j}$. Clearly, $w_{j} \in W_{0}^{1, p}(\Omega), w_{j} \rightarrow 0$ strongly in $L^{p}(\Omega)$ and

$$
\left\|\nabla w_{j}-\nabla u_{j}\right\|_{p ; \Omega} \leq\left(\int_{\Omega \backslash B_{0, \delta_{j}}}\left|\nabla u_{j}\right|^{p} d x\right)^{\frac{1}{p}}+2 \sqrt{\left\|u_{j}\right\|_{p ; \Omega}} .
$$

It follows that $u_{j}-w_{j} \rightarrow 0$ strongly in $W^{1, p}(\Omega)$. Because $\mathcal{C}_{c}^{\infty}(\Omega)$ is dense in $W_{0}^{1, p}(\Omega)$ the assertion of the lemma follows.

I am indebted to Stefan Müller for bringing the following result to my attention.

Lemma 3.2 Let $p \in[1, \infty)$ and let $\left\{V_{j}\right\}$ be a sequence in $L^{p}\left(\Omega ; \mathbf{R}^{m}\right)$. Then the following three assertions are equivalent:

(a) $\left\{\left|V_{j}\right|^{p}\right\}$ is uniformly summable on $\Omega$.

(b) For all $q>p$ and all $\varepsilon>0$ there exist $W_{j} \in L^{q}\left(\Omega ; \mathbf{R}^{m}\right)$, such that

$$
\sup _{j}\left\|W_{j}\right\|_{q ; \Omega}<\infty \text { and }\left\|V_{j}-W_{j}\right\|_{p ; \Omega} \leq \varepsilon .
$$

(c) For some $q>p$ and all $\varepsilon>0$ there exist $W_{j} \in L^{q}\left(\Omega ; \mathbf{R}^{m}\right)$, such that

$$
\sup _{j}\left\|W_{j}\right\|_{q ; \Omega}<\infty \text { and }\left\|V_{j}-W_{j}\right\|_{p ; \Omega} \leq \varepsilon .
$$

Proof. It follows easily by writing down the definitions.

Lemma 3.3 Suppose that $\Omega$ is a bounded Lipschitz domain and that $u_{j} \rightarrow 0$ weakly in $W^{1,1}(\Omega)$. Assume furthermore that for some $p \in[1, \infty)$ there is a sequence $\left\{V_{j}\right\}$ of vector fields in $L^{p}$ with the properties $V_{j}=0$ almost everywhere on $\mathbf{R}^{m} \backslash \Omega,\left\{\left|V_{j}\right|^{p}\right\}$ uniformly summable on $\Omega$ and $\nabla u_{j}-V_{j} \rightarrow 0$ in measure on $\Omega$. Then there exists a sequence $\left\{v_{j}\right\}$ in $\mathcal{C}_{c}^{\infty}(\Omega)$, such that

$$
\left\|v_{j}-u_{j}\right\|_{1,1 ; \Omega} \rightarrow 0 \text { and }\left\{\left|\nabla v_{j}\right|^{p}\right\} \text { is uniformly summable on } \Omega \text {. }
$$

Before we embark on the proof we recall some facts on the Hodge decomposition.

Let $L^{p}$ denote the Lebesgue space of all $p$-summable vector fields $V: \mathbf{R}^{m} \rightarrow \mathbf{R}^{m}$ and consider the subspaces $K^{p}$ and $H^{p}$ consisting of respectively the curl-free and the divergencefree vector fields, i.e.

$$
K^{p}=\left\{V \in L^{p}: \operatorname{curl} V=0\right\} \quad \text { and } \quad H^{p}=\left\{V \in L^{p}: \operatorname{div} V=0\right\} .
$$

It is readily seen that $K^{p}$ and $H^{p}$ are closed in $L^{p}$. If $u \in W_{l o c}^{1, p}\left(\mathbf{R}^{m}\right)$ and $\nabla u \in L^{p}$, then clearly $\nabla u \in K^{p}$ and it is not difficult to show that all vector fields in $K^{p}$ can be represented this way, i.e.

$$
K^{p}=\left\{\nabla u: u \in L_{l o c}^{p} \text { and } \nabla u \in L^{p}\right\} .
$$


The orthogonal complement of $K^{2}$ in $L^{2}$ is $H^{2}$. Therefore $L^{2}=K^{2} \oplus H^{2}$ by the Projection Theorem. Let $\mathbf{K}$ and $\mathbf{H}$ denote the corresponding orthogonal projections. We extend the Hodge decomposition $V=\mathbf{K}(V)+\mathbf{H}(V)$ to all $V \in L^{p}$ by showing that the operators $\mathbf{K}$ and $\mathbf{H}$ are of strong type $(p, p)$ for all $p \in(1, \infty)$.

The orthogonal projection of $L^{2}$ onto $K^{2}$ coincides with the metric projection onto $K^{2}$. Therefore $\mathbf{K}(V)=\nabla u_{V}$, where $u_{V} \in L^{1,2}\left(\mathbf{R}^{m}\right)$ is a minimiser of the functional

$$
\|\nabla u-V\|_{2}^{2}=\int_{\mathbf{R}^{m}}|\nabla u-V|^{2} d x, u \in L^{1,2}\left(\mathbf{R}^{m}\right) .
$$

By convexity it follows that $u_{V}$ is the (unique up to additive constants) solution of the Euler-Lagrange equation

$$
\operatorname{div} D u=\operatorname{div} V .
$$

Using the Fourier transformation, denoted by $\mathbf{F}$, we derive the formula

$$
\mathbf{K}(V)=\mathbf{F}^{-1}(M \mathbf{F} V),
$$

where $M(\xi)=-(\xi \otimes \xi) /|\xi|^{2}$. In view of the Mihlin Multiplier Theorem it follows that $\mathbf{K}$ is of strong type $(p, p)$ for all $p \in(1, \infty)$ and of weak type $(1,1)$.

Proof of Lemma 3.3. Only the case $p>1$ requires a proof. By Lemma 3.1 we can assume that $u_{j} \in W_{0}^{1, p}(\Omega)$ and therefore we may extend each $u_{j}$ to a function in $W^{1, p}\left(\mathbf{R}^{m}\right)$ by defining $u_{j}=0$ outside $\Omega$.

From the Dunford-Pettis Theorem we infer that $\left\{\nabla u_{j}\right\}$ is uniformly summable on $\Omega$. Also $\left\{V_{j}\right\}$ is uniformly summable on $\Omega$; hence applying Vitali's Convergence Theorem to the sequence $\left\{\nabla u_{j}-V_{j}\right\}$ on $\Omega$ we deduce $\left\|\nabla u_{j}-V_{j}\right\|_{1 ; \Omega} \rightarrow 0$. Since $\nabla u_{j}-V_{j}=0$ outside $\Omega$,

$$
\left\|\nabla u_{j}-V_{j}\right\|_{1} \rightarrow 0
$$

By (3.1) there are $w_{j} \in L_{l o c}^{p}$ with $\nabla w_{j} \in L^{p}$ and $\int_{\Omega} w_{j}=0$, such that $\nabla w_{j}=\mathbf{K}\left(V_{j}\right)$. Because $\sup _{j}\left\|V_{j}\right\|_{p}<\infty$ it follows from (3.2) that $V_{j} \rightarrow 0$ weakly in $L^{p}$ and since $\mathbf{K}$ is of strong type $(p, p), \nabla w_{j} \rightarrow 0$ weakly in $L^{p}$. Therefore, in view of Poincaré's inequality, $w_{j} \rightarrow 0$ weakly in $W^{1, p}(\Omega)$. Since $\nabla u_{j}-\nabla w_{j}=\nabla u_{j}-V_{j}+\mathbf{H}\left(V_{j}\right)$ and $\mathbf{H}\left(V_{j}\right)=\mathbf{H}\left(V_{j}-\nabla u_{j}\right)$ we infer from (3.2) and the weak type $(1,1)$ of $\mathbf{H}$ that, in particular,

$$
\nabla u_{j}-\nabla w_{j} \rightarrow 0 \text { in measure. }
$$

This together with uniform summability on $\Omega$ implies by Vitali's Convergence Theorem that $\left\|\nabla u_{j}-\nabla w_{j}\right\|_{1 ; \Omega} \rightarrow 0$. By the Rellich-Kondrachov Compactness Theorem we deduce that $\left\|u_{j}-w_{j}\right\|_{1,1 ; \Omega} \rightarrow 0$.

Next we show that $\left\{\left|\nabla w_{j}\right|^{p}\right\}$ is uniformly summable on $\Omega$. Let $q>p$ be fixed. Take $\varepsilon>0$. Since $\left\{\left|V_{j}\right|^{p}\right\}$ is uniformly summable on $\Omega$ we can by Lemma 3.2 find $W_{j}$ verifying

$$
\left\|V_{j}-W_{j}\right\|_{p ; \Omega} \leq \varepsilon \text { and } \sup _{j}\left\|W_{j}\right\|_{q ; \Omega}<\infty .
$$

Recall that $V_{j}=0$ outside $\Omega$ and define likewise $W_{j}=0$ outside $\Omega$. Then clearly $\| V_{j}-$ $W_{j} \|_{p} \leq \varepsilon$ and consequently $\left\|\nabla w_{j}-\mathbf{K}\left(W_{j}\right)\right\|_{p} \leq c_{p} \varepsilon$ and $\sup _{j}\left\|\mathbf{K}\left(W_{j}\right)\right\|_{q} \leq c_{q} \sup _{j}\left\|W_{j}\right\|_{q}$. By Lemma 3.2 we infer that $\left\{\left|\nabla w_{j}\right|^{p}\right\}$ is uniformly summable on $\Omega$.

The proof is concluded by use of Lemma 3.1. 
Lemma 3.4 (K. Zhang, [60] Lemma 3.1.) Suppose that $\Omega$ is a bounded Lipschitz domain and that $u_{j} \rightarrow 0$ weakly in $W^{1,1}(\Omega)$. Assume furthermore that there is a sequence $\left\{V_{j}\right\}$ of vector fields in $L^{\infty}$ with the properties $V_{j}=0$ almost everywhere on $\mathbf{R}^{m} \backslash \Omega$, $\sup _{j}\left\|V_{j}\right\|_{\infty}<$ $\infty$ and $D u_{j}-V_{j} \rightarrow 0$ in measure on $\Omega$. Then there exists a sequence $\left\{v_{j}\right\}$ in $\mathcal{C}_{c}^{\infty}(\Omega)$, such that

$$
\left\|v_{j}-u_{j}\right\|_{1,1 ; \Omega} \rightarrow 0 \text { and } \sup _{j}\left\|v_{j}\right\|_{1, \infty ; \Omega}<\infty
$$

Remark. This statement is not identical to Lemma 3.1 in [60], but follows easily from its proof. Some subtle generalisations have been obtained recently in [46].

Proof of Lemma 1.7. The case $p<\infty$ is covered by Lemma 3.3 and the case $p=\infty$ by Lemma 3.4 .

With Lemma 1.7 at our disposal Corollaries 1.8 and 1.9 are easy to prove.

Proof of Corollary 1.8. It is clear that the underlying deformation $u$ belongs to $W^{1, p}\left(\Omega ; \mathbf{R}^{n}\right)$ and because $\Omega$ can be written as an increasing union of Lipschitz domains each compactly contained in $\Omega$ we can assume that $\Omega$ is a Lipschitz domain.

By assumption we may find a sequence $\left\{u_{j}\right\}$, such that $u_{j} \rightarrow u$ weakly in $W^{1,1}\left(\Omega ; \mathbf{R}^{n}\right)$ and $\left\{\nabla u_{j}\right\}$ generates $\nu$. Because $\left\{\nabla u_{j}-\nabla u\right\}$ generates the Young measure $\lambda=\int_{\Omega} \delta_{x} \otimes$ $\left(\nu_{x} \star \delta_{-\nabla u(x)}\right) d x$ we can without loss of generality assume that $u \equiv 0$.

Suppose first that $p<\infty$. Define for $t>0$ the mapping $T_{t}(X)=\min \{t,|X|\} X /|X|$ and notice that the sequence $\left\{\left|T_{t}\left(\nabla u_{j}\right)\right|^{p}\right\}$ is uniformly bounded. By Theorem 2.4 it follows that

$$
\lim _{j \rightarrow \infty} \int_{\Omega}\left|T_{t}\left(\nabla u_{j}\right)\right|^{p} d x=\int_{\Omega \times \mathbf{R}^{n \times m}}\left|T_{t}(X)\right|^{p} d \nu(x, X),
$$

and thus

$$
\lim _{t \rightarrow \infty} \lim _{j \rightarrow \infty} \int_{\Omega}\left|T_{t}\left(\nabla u_{j}\right)\right|^{p} d x \leq \int_{\Omega \times \mathbf{R}^{n \times m}}|X|^{p} d \nu(x, X) .
$$

We can therefore find $t_{j} \rightarrow \infty$, such that

$$
\lim _{j \rightarrow \infty} \int_{\Omega}\left|T_{t_{j}}\left(\nabla u_{j}\right)\right|^{p} d x \leq \int_{\Omega \times \mathbf{R}^{n \times m}}|X|^{p} d \nu(x, X)<\infty .
$$

Let $V_{j}=T_{t_{j}}\left(\nabla u_{j}\right)$ and notice that $V_{j}-\nabla u_{j} \rightarrow 0$ in measure on $\Omega$. By Lemma 2.3, $\left\{V_{j}\right\}$ generates the Young measure $\nu$ and therefore, in view of (3.3) and Theorem 2.4, $\left\{\left|V_{j}\right|^{p}\right\}$ is uniformly summable on $\Omega$. We conclude by applying Lemma 3.3 to each row in $\left\{\nabla u_{j}\right\}$ and by utilising Lemma 2.3 once again.

Assume next that $p=\infty$. Then there exists a $R>0$, such that for almost all $x$ the support of $\nu_{x}$ is contained in the ball $|X| \leq R$. Thus if we take $t>R$ and let $V_{j}=T_{t}\left(\nabla u_{j}\right)$ we have

$$
\limsup _{j \rightarrow \infty} \int_{\Omega}\left|\nabla u_{j}-V_{j}\right| d x=0
$$

and therefore the claim follows if we apply Lemma 3.4 to each row.

Proof of Corollary 1.9. Assume that $p \in(1, \infty)$. The cases $p \in\{1, \infty\}$ are left to the interested reader. 
Let $u$ denote an underlying deformation for $\nu$. Take a ball $B_{x, r} \subset \Omega$ and define the measure $\lambda$ as

$$
\lambda=\int_{B_{x, r}} \delta_{y} \otimes\left(\nu_{y} \star \delta_{-\nabla u(y)}\right) d y
$$

Clearly, $\lambda$ is a gradient $p$-Young measure (on $B_{x, r} \times \mathbf{R}^{n \times m}$ ), which has $v \equiv 0$ as an underlying deformation. Consequently, there is a sequence $\left\{v_{j}\right\} \subset \mathcal{C}_{c}^{\infty}\left(B_{x, r} ; \mathbf{R}^{n}\right)$ as in Corollary 1.8. Due to the growth condition (1.22), the sequence $\left\{f\left(\nabla u(x)+\nabla v_{j}\right)\right\}$ is uniformly summable on $B_{x, r}$ and therefore we obtain by Theorem 2.4 and the definition of $\lambda$

$$
\lim _{j \rightarrow \infty} \int_{B_{x, r}} f\left(\nabla u(x)+\nabla v_{j}\right) d y=\int_{B_{x, r}} \int f(X+\nabla u(x)-\nabla u(y)) d \nu_{y}(X) d y
$$

By quasiconvexity we have for all $j$

$$
\int_{B_{x, r}} f\left(\nabla u(x)+\nabla v_{j}\right) d y \geq \mathcal{L}^{m}\left(B_{x, r}\right) f(\nabla u(x)),
$$

and the conclusion now follows from Lebesgue's Differentiation Theorem.

\section{Approximation of quasiconvex functions}

This section contains a proof of Proposition 1.10. For the purpose of proving the lower semicontinuity results of Theorems 1.1 and 1.2 (in the case $1<p<\infty$ ) the important fact is that the approximating functions can be taken to be of linear growth at infinity. The refinement that they can be taken convex outside large balls is only used in the Appendix.

The proof is divided into two steps each formulated as a lemma. In the first step it is shown by use of Corollaries 1.8 and 1.9 that it is possible to approximate with special quasiconvex functions of linear growth at infinity. The next step uses Lemma 1.6 and concerns approximation of the special quasiconvex functions encountered in the first step. The desired approximation result follows from this.

Lemma 4.1 Let $f: \mathbf{R}^{n \times m} \rightarrow \mathbf{R}$ be a quasiconvex function, such that for some $c_{1}, c_{2}>0$ and $p>1$

$$
c_{1}|X|^{p}-c_{2} \leq f(X) \leq c_{2}\left(|X|^{p}+1\right)
$$

holds for all $X$. Then there exist $f_{j}: \mathbf{R}^{n \times m} \rightarrow \mathbf{R}$ that are quasiconvex and satisfy

(a) $f_{j}(X) \leq f_{j+1}(X)$

(b) $f_{j}(X) \rightarrow f(X)$,

(c) $\lim _{X \rightarrow \infty}\left(f_{j}(X) /|X|\right) \in \mathbf{R}$ for each $j$. 
Proof. We define $f_{j}$ as $f_{j}=F_{j}^{q c}$, where $F_{j}(X)=\min \{j(1+|X|), f(X)\}$. Notice that hereby $f_{j}$ are quasiconvex and satisfy conditions (a) and (c). It is also clear that $f_{j} \leq f$. By translation it suffices to show that $f_{j}(0) \rightarrow f(0)$. For that purpose we apply Lemma 2.7 and find $u_{j} \in W_{0}^{1, \infty}\left(B ; \mathbf{R}^{n}\right)$, where $B=\{x:|x|<1\}$, such that $\left\|u_{j}\right\|_{\infty ; B} \rightarrow 0$ and

$$
f_{j}(0)+\frac{1}{j}>f_{B} F_{j}\left(\nabla u_{j}(x)\right) d x .
$$

Let $t_{j} \in \mathbf{R}$ denote the right-hand side of (4.2). Extracting a subsequence if necessary we can assume that $t_{j} \rightarrow t \in \mathbf{R}$. The lower bound in (4.1) implies that $\left\{\nabla u_{j}\right\}$ is bounded in $L^{1}$ and hence that $\left\{u_{j}\right\}$ is bounded in $W_{0}^{1,1}\left(B ; \mathbf{R}^{n}\right)$. By Theorem 2.4 we can extract a subsequence (for convenience not relabelled), such that $\left\{\nabla u_{j}\right\}$ generates a Young measure $\nu$ on $B \times \mathbf{R}^{n \times m}$.

Put $E_{j}=\left\{x \in B: j\left(1+\left|\nabla u_{j}(x)\right|\right)<f\left(\nabla u_{j}(x)\right)\right\}$ and notice that

$$
t_{j} \mathcal{L}^{m}(B)=j \int_{E_{j}}\left(1+\left|\nabla u_{j}\right|\right) d x+\int_{B \backslash E_{j}} f\left(\nabla u_{j}\right) d x
$$

and therefore by (4.2) and the lower bound in (4.1),

$$
\int_{E_{j}}\left(1+\left|\nabla u_{j}\right|\right) d x \rightarrow 0 \text { and } \sup _{j} \int_{B \backslash E_{j}}\left|\nabla u_{j}\right|^{p} d x<\infty .
$$

By de la Vallée-Poussin's criterion $\left\{\nabla u_{j} 1_{B \backslash E_{j}}\right\}$ is uniformly summable on $B$ and since $\left|\nabla u_{j}\right| 1_{E_{j}} \rightarrow 0$ strongly in $L^{1}(B)$ it follows that $\left\{\nabla u_{j}\right\}$ is uniformly summable on $B$. This implies that $u_{j} \rightarrow 0$ weakly in $W_{0}^{1,1}\left(B ; \mathbf{R}^{n}\right)$ and that $\nu$ is a gradient 1 -Young measure. Passing to the limit in (4.2) yields by Theorem 2.4 (applied to the normal integrand $F(t, X)=t^{+} f(X)$ and the sequence $\left.\left(1_{B \backslash E_{j}}, \nabla u_{j}\right)\right)$

$$
\lim _{j \rightarrow \infty} f_{j}(0) \mathcal{L}^{m}(B) \geq \liminf _{j \rightarrow \infty} \int_{B \backslash E_{j}} f\left(\nabla u_{j}\right) d x \geq \int_{B \times \mathbf{R}^{n \times m}} f(X) d \nu(x, X) .
$$

Referring to the lower bound in (4.1) it follows that $\nu$ has a finite $p^{\text {th }}$ order moment and therefore by Corollary 1.8 that it is a gradient $p$-Young measure. The proof is concluded using Corollary 1.9 taking into account that an underlying deformation for $\nu$ is $u \equiv 0$.

Lemma 4.2 Let $f: \mathbf{R}^{n \times m} \rightarrow \mathbf{R}$ be a quasiconvex function, such that

$$
\lim _{X \rightarrow \infty} \frac{f(X)}{|X|}=1
$$

There exist $f_{j}: \mathbf{R}^{n \times m} \rightarrow \mathbf{R}$ that are quasiconvex and satisfy

(a) $f_{j}(X) \leq f_{j+1}(X)$,

(b) $f_{j}(X) \rightarrow f(X)$,

(c) $f_{j}(X)=f_{j}^{\star \star}(X)=a_{j}|X|+b_{j}$ for large $|X|$, where $a_{j}, b_{j} \in \mathbf{R}$. 
Proof. Since $f$ is bounded from below we can without loss of generality assume that $f$ is strictly positive. Fix $\delta \in(0,1)$ and take $c=c(\delta) \in \mathbf{R}$, such that

$$
f(X) \geq \delta|X|+c
$$

for all $X$. For each positive integer $k$ we define the function $g_{k}: \mathbf{R}^{n \times m} \rightarrow \mathbf{R}$ as $g_{k}=G_{k}^{q c}$, where

$$
G_{k}(X)=\left\{\begin{array}{cc}
f(X) & \text { if }|X| \leq k \\
\delta|X|+c & \text { if }|X|>k
\end{array}\right.
$$

Observe that $g_{k}$ is quasiconvex, satisfies $g_{k}(X)=\delta|X|+c$ if $|X|>k$ and $g_{k}(X) \leq$ $g_{k+1}(X) \leq f(X)$ for all $X$ and all $k$.

Fix $X \in \mathbf{R}^{n \times m}$. We claim that

$$
\lim _{k \rightarrow \infty} g_{k}(X) \geq\left(2-\frac{1}{\delta}\right) f(X)
$$

Since $g_{k}(X)=\inf \left\{f_{B} G_{k}(X+\nabla u) d x: u \in W_{0}^{1, \infty}\left(B ; \mathbf{R}^{n}\right)\right\}$ and $G_{k}(X) \geq \delta|X|+c$ for all $X$ and $k$ there exists by Lemma 2.7 a sequence $\left\{u_{k}\right\} \subset W_{0}^{1, \infty}\left(B ; \mathbf{R}^{n}\right)$ satisfying

$$
\begin{gathered}
\left(g_{k}(X)+\frac{1}{k}\right) \mathcal{L}^{m}(B)>\int_{B} G_{k}\left(X+\nabla u_{k}\right) d x, \\
u_{k} \rightarrow 0 \text { strongly in } L^{1}\left(B ; \mathbf{R}^{n}\right) \text { and } \sup _{k} \int_{B}\left|\nabla u_{k}\right| d x<\infty .
\end{gathered}
$$

If $E_{k}=\left\{x \in B:\left|X+\nabla u_{k}(x)\right|>k\right\}$, then

$$
\lim _{k \rightarrow \infty} g_{k}(X) \mathcal{L}^{m}(B) \geq \limsup _{k \rightarrow \infty} \int_{B} f\left(X+\nabla u_{k}\right) d x-(1-\delta) \liminf _{k \rightarrow \infty} \int_{E_{k}}\left|X+\nabla u_{k}\right| d x
$$

Since $\lim \inf _{k \rightarrow \infty} \int_{E_{k}}\left|X+\nabla u_{k}\right| d x \leq \lim \sup _{k \rightarrow \infty} \int_{B} f\left(X+\nabla u_{k}\right) / \delta d x$ and since by Lemma 2.8 it follows that

$$
\limsup _{k \rightarrow \infty} \int_{B} f\left(X+\nabla u_{k}\right) d x \geq f(X) \mathcal{L}^{m}(B)
$$

the inequality (4.4) follows.

Next take for a positive integer $j, \delta=1-1 / j$ and consider the corresponding sequence $\left\{g_{j, k}\right\}_{k=1}^{\infty}$ as constructed above. For each $j$, there exists by (4.4) and Dini's Lemma an integer $k_{j}$, such that

$$
g_{j, k_{j}}(X)>\left(1-\frac{2}{j-1}\right) f(X) \text { if }|X| \leq j .
$$

If we define $f_{j}=\max \left\{g_{i, k_{i}}: i=1, \ldots, j\right\}$, then $f_{j}$ are quasiconvex and satisfy (a)-(c). Indeed, (a) and (b) are obvious from the construction and (c) follows because

$$
f_{j}(X) \geq \max _{1 \leq i \leq j}\left\{(1-1 / i)|X|+c_{i}\right\}
$$

with equality if $|X| \geq \max _{1 \leq i \leq j} k_{i}$.

Proof of Proposition 1.10. This is a straightforward consequence of Lemma 4.1 and Lemma 4.2 . 


\section{$5 \quad$ Lower semicontinuity in Sobolev-type spaces}

The principal goals in the section are the proofs of Theorems 1.1 and 1.3. We start with an elementary lemma.

Lemma 5.1 Let $\mu$ and $\nu$ be non-negative, finite Radon measures on $\Omega$ and let $\left\{\lambda_{j}\right\}$ be a sequence of bounded $\mathbf{R}^{d}$-valued Radon measures on $\Omega$. If $\left\{\lambda_{j}\right\}$ is uniformly $\mu-A C$ and if

$$
\lambda_{j}=V_{j} \cdot \nu+\lambda_{j}^{s},
$$

denotes the Lebesgue-Radon-Nikodym decomposition of $\lambda_{j}$ with respect to $\nu$, then $\left\{V_{j}\right\}$ is uniformly $\nu$ summable.

Proof. This is easily seen by writing down the definitions.

Lemma 5.2 Let $\mu$ be a non-negative, finite Radon measure on $\Omega$ and let $\left\{u_{j}\right\}$ be a sequence in $B V\left(\Omega ; \mathbf{R}^{n}\right)$. Assume that $\left\{D u_{j}\right\}$ is uniformly $\mu-A C$ and that $\left\{\nabla u_{j}\right\}$ generates the Young measure $\nu$. Then there exists a $\mathcal{L}^{m}$ negligible set $N \subset \Omega$, such that for $x \in \Omega \backslash N$ each $\nu_{x}$ is a probability measure with a centre of mass $\bar{\nu}_{x}$ and Jensen's inequality

$$
\int f d \nu_{x} \geq f\left(\bar{\nu}_{x}\right)
$$

holds for all quasiconvex functions $f: \mathbf{R}^{n \times m} \rightarrow \mathbf{R}$ for which $f(X) /|X|$ has a finite limit as $X \rightarrow \infty$.

Proof. The proof proceeds in four steps.

1. Claim: without loss of generality we can assume that $\sup _{j}\left|D u_{j}\right|(\Omega)<\infty$ and that $u_{j} \rightarrow u$ strongly in $L^{1}\left(\Omega ; \mathbf{R}^{n}\right)$ for some $u \in B V\left(\Omega ; \mathbf{R}^{n}\right)$.

Since $m>1$ and $\left|D u_{j}\right|(A)=0$ whenever $\mathcal{H}^{m-1}(A)=0$ it follows that $\left\{D u_{j}\right\}$ is uniformly $\mu_{n a}$-AC, where $\mu_{n a}$ is the non-atomic part of $\mu$, i.e. $\mu_{n a}=\mu-\sum_{x \in \Omega} \mu(\{x\}) \delta_{x}$. Henceforth we shall suppose that $\mu=\mu_{n a}$ and the uniform $\mu$-AC, then implies that $\sup _{j}\left|D u_{j}\right|(\Omega)<$ $\infty$. Let $B$ be an open ball contained in $\Omega$ and define

$$
v_{j}(x)=u_{j}(x)-f_{B} u_{j}(y) d y, x \in B .
$$

Clearly, $D v_{j}=D u_{j}\left\lfloor B\right.$ and by Poincaré's inequality it follows that $\left\{v_{j}\right\}$ is bounded in $B V\left(B ; \mathbf{R}^{n}\right)$. By virtue of the Rellich-Kondrachov Compactness Theorem there is a subsequence (for convenience not relabelled) and some $v \in B V\left(B ; \mathbf{R}^{n}\right)$, such that $v_{j} \rightarrow v$ strongly in $L^{1}\left(B ; \mathbf{R}^{n}\right)$. Note that $\left\{\nabla v_{j}\right\}$ generates the Young measure $\nu\left\lfloor\left(B \times \mathbf{R}^{n \times m}\right)=\right.$ $\int_{B} \delta_{x} \otimes \nu_{x} d x$. Since it is sufficient to prove that the Young measure $\nu$ has the claimed property on any ball contained in $\Omega$ Step 1 concluded.

2. Consider the Lebesgue-Radon-Nikodym decompositions with respect to the Lebesgue measure:

$$
\mu=a \cdot \mathcal{L}^{m}+\mu^{s} \text { and } D u_{j}=\nabla u_{j} \cdot \mathcal{L}^{m}+V_{j} \cdot \mu^{s} .
$$


Claim: without loss of generality we can assume that $u \equiv 0, \nabla u_{j} \rightarrow 0$ weakly in $L^{1}\left(\Omega ; \mathbf{R}^{n \times m}\right)$ and $V_{j} \rightarrow 0$ weakly in $L_{\mu^{s}}^{1}\left(\Omega ; \mathbf{R}^{n \times m}\right)$.

By Lemma 5.1, $\left\{\nabla u_{j}\right\}$ is uniformly $\mathcal{L}^{m}$ summable on $\Omega$ and $\left\{V_{j}\right\}$ is uniformly $\mu^{s}$ summable on $\Omega$. Furthermore we deduce from Step 1 that $D u_{j} \rightarrow D u$ weakly* in $\mathcal{C}_{0}^{0}(\Omega)^{\prime}$ and using the uniform summability of the terms in the Lebesgue decompositions we deduce

$$
\nabla u_{j} \rightarrow \nabla u \text { weakly in } L^{1}\left(\Omega ; \mathbf{R}^{n \times m}\right)
$$

and

$$
V_{j} \rightarrow V \text { weakly in } L_{\mu^{s}}^{1}\left(\Omega ; \mathbf{R}^{n \times m}\right) .
$$

The claim follows by considering the difference $\left\{u_{j}-u\right\}$, using the observation made at the end of Subsection 2.2 and noting that the class of quasiconvex functions $f$ for which $f(X) /|X|$ has a finite limit as $X \rightarrow \infty$ is invariant under translation.

3. Fix a quasiconvex function $f: \mathbf{R}^{n \times m} \rightarrow \mathbf{R}$ with the property $f(X) /|X| \rightarrow 1$ as $X \rightarrow \infty$.

Claim: there exists a negligible set $N_{f} \subset \Omega$, such that for $x \in \Omega \backslash N_{f} \nu_{x}$ is a probability measure, $\bar{\nu}_{x}=0$ and

$$
\int f d \nu_{x} \geq f(0)
$$

Let $\Omega_{0}$ be the set of all $x$ in $\Omega$ for which $\nu_{x}$ is a probability measure with $\bar{\nu}_{x}=0$. The set $\Omega_{0}$ has full measure in $\Omega$. Fix $x \in \Omega_{0}$ and take $r>0$, such that $B_{x, r} \subset \Omega$. In view of (1.21) with $X=0, v=0$ and $\delta \in(0,1)$ we have

$$
\begin{gathered}
f(0) \mathcal{L}^{m}\left(B_{x, \delta r}\right) \leq \int_{B_{x, r}} f\left(\nabla u_{j}\right) d y+ \\
+\left|D^{s} u_{j}\right|\left(D^{s} u_{j}\right)+\frac{1}{(1-\delta) r} \int_{B_{x, r} \backslash B_{x, \delta r}}\left|u_{j}(y)\right| d y
\end{gathered}
$$

Since $\left\{f\left(\nabla u_{j}\right)\right\}$ is uniformly summable and $\left\{\nabla u_{j}\right\}$ generates the Young measure $\nu$ it follows from Theorem 2.4 that

$$
\int_{B_{x, r}} f\left(\nabla u_{j}\right) d y \rightarrow \int_{B_{x, r}} \int f d \nu_{y} d y
$$

For the singular measure we notice that

$$
\left|D^{s} u_{j}\right|\left(B_{x, r}\right)=\int_{B_{x, r}}\left|V_{j}\right| d \mu^{s}
$$

and since $\left\{\left|V_{j}\right|\right\}$ is uniformly $\mu^{s}$ summable and $\mu^{s}$ is non-atomic there exist by the DunfordPettis Theorem a subsequence $\left\{\left|V_{j_{k}}\right|\right\}$ and a $g \in L_{\mu^{s}}^{1}(\Omega)$, such that $\left|V_{j_{k}}\right| \rightarrow g$ weakly in $L_{\mu^{s}}^{1}(\Omega)$. Passing to the limit through this subsequence we obtain

$$
\delta^{m} f(0) \leq f_{B_{x, r}} \int f d \nu_{y} d y+\frac{1}{\mathcal{L}^{m}\left(B_{x, r}\right)} \int_{B_{x, r}} g d \mu^{s} .
$$


By Lebesgue's Differentiation Theorem the set $\Omega_{f}$ of points $x \in \Omega_{0}$ for which

$$
\mathcal{L}^{m}\left(B_{x, r}\right)^{-1} \int_{B_{x, r}} g d \mu^{s} \rightarrow 0
$$

and

$$
f_{B_{x, r}} \int f d \nu_{y} d y \rightarrow \int f d \nu_{x}
$$

as $r \rightarrow 0^{+}$has full $\mathcal{L}^{m}$ measure. If we define $N_{f}=\Omega \backslash \Omega_{f}$, then for $x \in \Omega \backslash N_{f}$ we obtain as $r \rightarrow 0^{+}$and then $\delta \rightarrow 1^{-}$that

$$
f(0) \leq \int f d \nu_{x}
$$

as claimed.

4. We show that it is possible to find a negligible set $N$, which is independent of $f$.

Let $\mathcal{E}$ denote the space of continuous functions $g: \mathbf{R}^{n \times m} \rightarrow \mathbf{R}$ for which $g(X) /|X|$ has a finite limit as $X \rightarrow \infty$ and define the norm of $g \in \mathcal{E}$ as

$$
\|g\|=\sup _{X} \frac{|g(X)|}{1+|X|}
$$

Hereby $\mathcal{E}$ is a separable Banach space and if therefore $\mathcal{Q}$ denotes the set of all quasiconvex functions in $\mathcal{E}$ there exists a countable set $F \subset \mathcal{Q}$, which is dense in $\mathcal{Q}$. We now define $N=\bigcup_{f \in F} N_{f}$ and it is not difficult to show that $N$ has the desired properties.

Proof of Theorem 1.3. By Lemma 5.2 there is a negligible set $N$, such that for all $x \in \Omega \backslash N$ each $\nu_{x}$ is a probability measure with a centre of mass and with the property that Jensen's inequality

$$
\int f d \nu_{x} \geq f\left(\bar{\nu}_{x}\right)
$$

holds for all quasiconvex functions $f$ for which $f(X) /|X|$ has a finite limit as $X \rightarrow \infty$. Since $\sup _{j}\left\|\nabla u_{j}\right\|_{p ; \Omega}<\infty$ it follows that for $p<\infty$,

$$
\int_{\Omega} \int|X|^{p} d \nu_{x}(X) d x<\infty
$$

and hence that

$$
\int|X|^{p} d \nu_{x}(X)<\infty
$$

for almost all $x$. For $p=\infty$ it follows that there exists a compact set $K \subset \mathbf{R}^{n \times m}$, such that for almost all $x$

$$
\nu_{x} \text { is supported in } K \text {. }
$$

Let $M \subset \Omega$ denote the exceptional set in (5.2) in case $p<\infty$ and in (5.3) in case $p=\infty$. The proof is concluded since for $x \in \Omega \backslash(N \cup M)$ it follows from Lemma 1.5 and Corollary 1.8 that $\left(\mathcal{L}^{m}\lfloor\Omega) \otimes \nu_{x}\right.$ is a gradient $p$-Young measure. 
Proof of Theorem 1.1. As mentioned in the Introduction it is possible to give a proof based on Theorem 1.3 and Theorem 2.4. We also mentioned that in the case $p \in(1, \infty)$ it is possible to give a proof, which only uses Proposition 1.10 and Lemma 5.2. We focus on the latter leaving the details of the case $p \in\{1, \infty\}$ to the interested reader.

We can assume that the left-hand side of $(1.7)$ is less than $\infty$. Because $\left\{F^{-}\left(x, u_{j}, \nabla u_{j}\right)\right\}$ is assumed uniformly summable it then follows that the left-hand side of (1.7) is a real number. By extracting a subsequence if necessary we can assume that

$$
\int_{\Omega} F\left(x, u_{j}, \nabla u_{j}\right) d x \rightarrow l \in \mathbf{R}
$$

Put $V_{j}=\left(u_{j}, \nabla u_{j}\right)$ and consider the corresponding sequence $\left\{\varepsilon_{V_{j}}\right\}$ of elementary Young measures. Since $\sup _{j} \mathcal{L}^{m}\left(\left\{x \in \Omega:\left|V_{j}(x)\right| \geq t\right\}\right) \rightarrow 0$ as $t \rightarrow \infty$ there exists by Lemma 2.2 a subsequence (for convenience not relabelled), which generates a Young measure $\lambda$. Because $u_{j} \rightarrow u$ locally in measure it follows that if

$$
\lambda=\int_{\Omega} \delta_{x} \otimes \lambda_{x} d x
$$

then $\lambda_{x}=\delta_{u(x)} \otimes \nu_{x}$, where

$$
\nu=\int_{\Omega} \delta_{x} \otimes \nu_{x} d x
$$

is a Young measure generated by $\left\{\nabla u_{j}\right\}$. In view of Theorem 2.4

$$
\int_{\Omega} \int F(x, u(x), X) d \nu_{x}(X) d x \leq l .
$$

As in the proof of Lemma 5.2 we see that $\nabla u_{j} \rightarrow \nabla u$ weakly in $L^{1}\left(\Omega ; \mathbf{R}^{n \times m}\right)$ and therefore $\bar{\nu}_{x}=\nabla u(x)$ almost everywhere. Let $N$ be the negligible set from Lemma 5.2 and put $\Omega^{\prime}=\left\{x \in \Omega \backslash N: \bar{\nu}_{x}=\nabla u(x)\right\}$. We claim that for $x \in \Omega^{\prime}$ the inequality

$$
F(x, u(x), \nabla u(x)) \leq \int F(x, u(x), X) d \nu_{x}(X)
$$

holds. Together with (5.4) this entails (1.7).

Fix $x \in \Omega^{\prime}$ and put $f(X)=F(x, u(x), X)$. Then $f$ is quasiconvex and by $\left(\mathrm{H}_{p}\right)$ and Lemma 2.5

$$
\limsup _{X \rightarrow \infty} \frac{|f(X)|}{|X|^{p}}<\infty
$$

For an integer $k>0$ define $f_{k}(X)=|X|^{p} / k+\max \{f(X),-k\}$. We now apply Proposition 1.10 to $f_{k}$ and we apply Lemma 5.2 to each of the approximating quasiconvex functions. Hereby we deduce that

$$
f_{k}(\nabla u(x)) \leq \int f_{k} d \nu_{x}=\frac{1}{k} \int|X|^{p} d \nu_{x}(X)+\int \max \{f,-k\} d \nu_{x}
$$

for all $\mathrm{k}$. Passing to the limit $k \rightarrow \infty$ the inequality (5.5) follows. 


\section{Lower semicontinuity in GSBV}

Before proceeding with the proof of Theorem 1.4 we briefly recall some definitions and results from [6]. The following definition is motivated by some minimisation problems that are not coercive in $S B V\left(\Omega ; \mathbf{R}^{n}\right)$, but in the enlargement $G S B V\left(\Omega ; \mathbf{R}^{n}\right)$ (see [6], Example $5.3)$.

Definition (L. Ambrosio and E. De Giorgi, [10].) A Borel function $u: \Omega \rightarrow \mathbf{R}^{n}$ is a generalised special function of bounded variation, briefly $u \in G S B V\left(\Omega ; \mathbf{R}^{n}\right)$, if $\varphi \circ u \in$ $S B V_{l o c}(\Omega)$ for all $\varphi \in \mathcal{C}^{1}\left(\mathbf{R}^{n}\right)$ for which $\nabla \varphi$ has compact support.

Remarks. 1. Under the natural definitions of 'addition' and 'multiplication with scalar' the space $G S B V\left(\Omega ; \mathbf{R}^{n}\right)$ is a vector space.

2. For bounded functions there is nothing new as $G S B V \cap L^{\infty}=S B V_{l o c}$.

3. In this paper we assume that $n>1$ and then we have that a Borel function $u \in$ $G S B V\left(\Omega ; \mathbf{R}^{n}\right)$ if and only if $\varphi \circ u \in S B V_{l o c}(\Omega)$ for all $\varphi \in \mathcal{C}_{c}^{1}\left(\mathbf{R}^{n}\right)$. Recall that $v \in$ $S B V_{\text {loc }}(\Omega)$ if and only if $\left.v\right|_{\omega} \in S B V(\omega)$ for all open subsets $\omega$ that are compactly contained in $\Omega$.

Proposition 6.1 (L. Ambrosio, [6], Propositions 1.3 and 1.4.) Let $u \in G S B V\left(\Omega ; \mathbf{R}^{n}\right)$. Then $u$ is approximately differentiable almost everywhere in $\Omega$ and the jump set $S_{u}$ is countably rectifiable. If $N_{u}$ denotes a Borel measurable unit normal to $S_{u}$, then it is possible to define one-sided traces $u^{+}$and $u^{-}$by the procedure described in Section 2.

Remark. If $u \in G S B V\left(\Omega ; \mathbf{R}^{n}\right)$ and $\varphi \in \mathcal{C}_{c}^{1}\left(\mathbf{R}^{n}\right)$, then

$$
D(\varphi \circ u)=\nabla \varphi(u) \nabla u \cdot \mathcal{L}^{m}\left\lfloor\Omega+\left(\varphi\left(u^{+}\right)-\varphi\left(u^{-}\right)\right) N_{u} \cdot \mathcal{H}^{m-1}\left\lfloor S_{u} .\right.\right.
$$

(Indeed, by the Chain Rule for approximate derivatives $\nabla(\varphi \circ u)(x)=\nabla \varphi(u(x)) \nabla u(x)$ and, since $\varphi$ especially is continuous, $\varphi(u)^{+}=\varphi\left(u^{+}\right)$on $S_{\varphi(u)} \subseteq S_{u}$ and $\varphi\left(u^{+}\right)-\varphi\left(u^{-}\right)=0$ on $\left.S_{u} \backslash S_{\varphi(u)}\right)$

The motivation for introducing the space $G S B V\left(\Omega ; \mathbf{R}^{n}\right)$ comes from the following compactness result. To state it we need three test functions $\phi, \theta$ and $g$.

Let $\phi:[0, \infty) \rightarrow[0, \infty]$ be a convex non-decreasing function satisfying the condition

$$
\frac{\phi(t)}{t} \rightarrow \infty \text { as } t \rightarrow \infty
$$

Let $\theta:[0, \infty] \rightarrow[0, \infty]$ be a concave non-decreasing function satisfying the condition

$$
\frac{\theta(t)}{t} \rightarrow \infty \text { as } t \rightarrow 0^{+}
$$

Let $g: \Omega \times \mathbf{R}^{n} \rightarrow[0, \infty]$ be a normal integrand satisfying the condition

$$
g(x, v) \rightarrow \infty \text { as } v \rightarrow \infty
$$

for almost all $x \in \Omega$. 
Theorem 6.2 (L. Ambrosio, [6] Theorem 2.2.) Let $\phi, \theta$ and $g$ be three functions as described above. Let $\left\{u_{j}\right\}$ be a sequence in $G S B V\left(\Omega ; \mathbf{R}^{n}\right)$ and assume that

$$
\sup _{j}\left\{\int_{\Omega}\left(g\left(x, u_{j}\right)+\phi\left(\left|\nabla u_{j}\right|\right)\right) d x+\int_{S_{u_{j}}} \theta\left(\left|u_{j}^{+}-u_{j}^{-}\right|\right) d \mathcal{H}^{m-1}\right\}<\infty .
$$

Then there exists a subsequence $\left\{u_{j_{k}}\right\}$ and a function $u \in G S B V\left(\Omega ; \mathbf{R}^{n}\right)$, such that $u_{j_{k}} \rightarrow$ $u$ in measure on $\Omega$ and $\nabla u_{j_{k}} \rightarrow \nabla u$ weakly in $L^{1}\left(\Omega ; \mathbf{R}^{n \times m}\right)$. Furthermore, the function $u$ also satisfies the inequality (6.4).

Remarks. 1. The last statement that $u$ also satisfies (6.4) is not explicit in [6]. However, it follows from the results in Section 5.1 of [6] that the integrand $\varphi(a, b, N)=\theta(|a-b|)$ is regularly biconvex and then the proof of Theorem 3.7 in [6] can be used to show that

$$
\int_{S_{u}} \theta\left(\left|u^{+}-u^{-}\right|\right) d \mathcal{H}^{m-1} \leq \liminf _{j \rightarrow \infty} \int_{S_{u_{j}}} \theta\left(\left|u_{j}^{+}-u_{j}^{-}\right|\right) d \mathcal{H}^{m-1} .
$$

The lower semicontinuity of the bulk energy term follows from Fatou's Lemma and the convexity of $X \mapsto \phi(|X|)$.

2. The function $\theta$ is sub-additive. (This follows easily if we for fixed $s \geq 0$ consider the auxiliary function $h(t)=\theta(t)+\theta(s)-\theta(s+t), t \geq 0$ and observe that it is non-decreasing because $\theta$ is concave.)

The main result of this section ensures (by (1.15) and Corollary 1.9) lower semicontinuity of quasiconvex integrals in the setting prescribed by this compactness result.

Theorem 6.3 Let $\left\{u_{j}\right\}$ be a sequence in $G S B V\left(\Omega ; \mathbf{R}^{n}\right)$, which satisfies the boundedness condition (6.4). Assume that for some $p \in[1, \infty], \sup _{j}\left\|\nabla u_{j}\right\|_{p ; \Omega}<\infty$ and that $\left\{\nabla u_{j}\right\}$ generates the Young measure

$$
\nu=\int_{\Omega} \delta_{x} \otimes \nu_{x} d x
$$

Then for almost all $x$ the measure $\left(\mathcal{L}^{m}\lfloor\Omega) \otimes \nu_{x}\right.$ is a gradient $p$-Young measure.

Remark. We obtain Theorem 1.4 in the special case, where $\left\{u_{j}\right\} \subset S B V\left(\Omega ; \mathbf{R}^{n}\right)$ and $g(x, v)=|v|$.

A brief outline of the proof is as follows. First it is shown, utilising Theorem 6.2, that it is not restrictive to assume that $u_{j} \rightarrow 0$ in measure and that $\nabla u_{j} \rightarrow 0$ weakly in $L^{1}$. Next we truncate the functions $u_{j}$ to obtain new functions $v_{j}$ with the properties $v_{j} \rightarrow 0$ in $L^{\infty}, \nabla u_{j}-\nabla v_{j} \rightarrow 0$ in $L^{1}$ and, by use of the Vitali-Hahn-Saks Theorem (Theorem 6.4 below), $\left\{D v_{j}\right\}$ is uniformly $\mu$-AC for some $\mu$. We conclude using Theorem 1.3.

Theorem 6.4 ([22], Theorem 2 p. 158.) Let $\mu$ be a non-negative, finite Radon measure on $\Omega$. Assume that $\left\{\lambda_{j}\right\}$ is a sequence of bounded $\mathbf{R}^{d}$-valued Radon measures on $\Omega$ and that each $\lambda_{j}$ is $\mu$-AC. If for all $\mu$ measurable sets $A \subseteq \Omega$ the limit $\lim _{j \rightarrow \infty} \lambda_{j}(A)$ exists in $\mathbf{R}^{d}$, then $\left\{\lambda_{j}\right\}$ is uniformly $\mu-A C$. 
Proof of Theorem 6.3. The proof proceeds in three steps.

1. It is not restrictive to assume that $u_{j} \rightarrow 0$ in measure on $\Omega$ and $\nabla u_{j} \rightarrow 0$ weakly in $L^{1}\left(\Omega ; \mathbf{R}^{n \times m}\right)$.

By Theorem 6.2 there exist a subsequence of $\left\{u_{j}\right\}$ (for convenience not relabelled) and $u \in G S B V\left(\Omega ; \mathbf{R}^{n}\right)$, such that $u_{j} \rightarrow u$ in measure on $\Omega$ and $\nabla u_{j} \rightarrow \nabla u$ weakly in $L^{1}\left(\Omega ; \mathbf{R}^{n \times m}\right)$. Define $v_{j}=u_{j}-u$. Then $v_{j} \in G S B V\left(\Omega ; \mathbf{R}^{n}\right), v_{j} \rightarrow 0$ in measure and $\nabla v_{j} \rightarrow 0$ weakly in $L^{1}\left(\Omega ; \mathbf{R}^{n \times m}\right)$. By the remarks following Theorem 6.2 and since $S_{v_{j}} \subseteq S_{u_{j}} \cup S_{u}$

$$
\sup _{j} \int_{S_{v_{j}}} \theta\left(\left|v_{j}^{+}-v_{j}^{-}\right|\right) d \mathcal{H}^{m-1} \leq 2 \sup _{j} \int_{S_{u_{j}}} \theta\left(\left|u_{j}^{+}-u_{j}^{-}\right|\right) d \mathcal{H}^{m-1} .
$$

This concludes Step 1.

2. Let $\varphi \in \mathcal{C}^{1}([0, \infty))$ be such that $\varphi(t)=t$ if $t \in[0,1]$ and $\varphi(t)=0$ if $t \geq 2$. Denote by $\operatorname{Lip}(\varphi)$ its Lipschitz constant and notice that $\operatorname{Lip}(\varphi) \in(1, \infty)$. Define the radial mapping

$$
\Phi(v)=\left\{\begin{array}{cl}
\varphi(|v|) \frac{v}{|v|} & \text { if } v \neq 0 \\
0 & \text { if } v=0
\end{array}\right.
$$

For $\delta>0$ define the rescaled function $\Phi_{\delta}(v)=\delta \Phi\left(\delta^{-1} v\right)$. Clearly, $\Phi_{\delta} \in \mathcal{C}_{c}^{1}\left(\mathbf{R}^{n} ; \mathbf{R}^{n}\right)$ and

$$
\operatorname{Lip}\left(\Phi_{\delta}\right)=\operatorname{Lip}(\varphi), \Phi_{\delta}(v)=v \text { if }|v| \leq \delta \text { and } \Phi_{\delta}(v)=0 \text { if }|v| \geq 2 \delta .
$$

Define $u_{j, \delta}=\Phi_{\delta}\left(u_{j}\right)$.

Claim: there exist numbers $\delta_{j}>0$, such that $\left\{u_{j, \delta_{j}}\right\} \subset S B V\left(\Omega ; \mathbf{R}^{n}\right)$ satisfies

$$
\begin{gathered}
\left\|u_{j, \delta_{j}}\right\|_{\infty ; \Omega} \rightarrow 0, \\
\left\|\nabla u_{j, \delta_{j}}-\nabla u\right\|_{1 ; \Omega} \rightarrow 0
\end{gathered}
$$

and

$$
D u_{j, \delta_{j}}(A) \rightarrow 0
$$

for all Borel sets $A \subseteq \Omega$.

Clearly, $u_{j, \delta} \in S B V_{l o c}\left(\Omega ; \mathbf{R}^{n}\right)$,

$$
\left\|u_{j, \delta}\right\|_{\infty ; \Omega} \leq \sup \left|\Phi_{\delta}\right| \leq \sup |\varphi| \delta
$$

and

$$
D u_{j, \delta}=\nabla u_{j, \delta} \cdot \mathcal{L}^{m}+\left(u_{j, \delta}^{+}-u_{j, \delta}^{-}\right) \otimes N_{u_{j, \delta}} \cdot \mathcal{H}^{m-1}\left\lfloor S_{u_{j, \delta}}\right.
$$

By the Chain Rule for approximate derivatives $\nabla u_{j, \delta}=\nabla \Phi_{\delta}\left(u_{j}\right) \nabla u_{j}$, where $(I$ denotes the $n \times n$ unit matrix)

$$
\nabla \Phi_{\delta}\left(u_{j}\right)=\nabla \Phi\left(\delta^{-1} u_{j}\right)=\frac{\varphi\left(\delta^{-1}\left|u_{j}\right|\right)}{\delta^{-1}\left|u_{j}\right|} I+\frac{u_{j} \otimes u_{j}}{\delta^{-1}\left|u_{j}\right|^{3}}\left[\delta^{-1}\left|u_{j}\right| \varphi^{\prime}\left(\delta^{-1}\left|u_{j}\right|\right)-\varphi\left(\delta^{-1}\left|u_{j}\right|\right)\right]
$$


In particular, $\nabla \Phi_{\delta}\left(u_{j}\right)=I$ when $\left|u_{j}\right| \leq \delta$, and by Step 1 we then deduce that

$$
\nabla u_{j, \delta}-\nabla u_{j} \rightarrow 0 \text { in measure on } \Omega \text {. }
$$

Since also $\left|\nabla u_{j, \delta}\right| \leq \operatorname{Lip}(\varphi)\left|\nabla u_{j}\right|$ it follows that $\left\{\nabla u_{j, \delta}-\nabla u_{j}\right\}$ is uniformly summable on $\Omega$ and consequently, by Vitali's Convergence Theorem,

$$
\lim _{j \rightarrow \infty}\left\|\nabla u_{j, \delta}-\nabla u_{j}\right\|_{1 ; \Omega}=0 .
$$

Put

$$
M=\sup _{j} \int_{S_{u_{j}}} \theta\left(\left|u_{j}^{+}-u_{j}^{-}\right|\right) d \mathcal{H}^{m-1} .
$$

By (6.4), $M<\infty$. Since $\Phi_{\delta}$ is continuous

$$
u_{j, \delta}^{+}=\Phi_{\delta}\left(u_{j}^{+}\right), u_{j, \delta}^{-}=\Phi_{\delta}\left(u_{j}^{-}\right) \text {and } S_{u_{j, \delta}} \subseteq S_{u_{j}},
$$

and, in particular, $\left|u_{j, \delta}^{+}-u_{j, \delta}^{-}\right| \leq c \delta$, where $c=2 \sup |\varphi|$. Next note that $\bar{\theta}(t)=\inf \left\{\theta(t), t^{\gamma}\right\}$, $\gamma \in(0,1)$, has the same properties as $\theta$ besides being 0 at $t=0$. Hence we can assume that $\theta(0)=0$. Because $\theta$ is concave the difference quotient $\theta(t) / t$ is non-increasing, hence

$$
\frac{\left|u_{j, \delta}^{+}-u_{j, \delta}^{-}\right|}{\theta\left(\left|u_{j, \delta}^{+}-u_{j, \delta}^{-}\right| / \operatorname{Lip}(\varphi)\right)} \leq \operatorname{Lip}(\varphi) \frac{c \delta}{\theta(c \delta)}
$$

$\mathcal{H}^{m-1}$ almost everywhere on $S_{u_{j, \delta}}$. Because $\theta$ is non-decreasing and $\operatorname{Lip}\left(\Phi_{\delta}\right)=\operatorname{Lip}(\varphi)$

$$
\theta\left(\frac{\left|u_{j, \delta}^{+}-u_{j, \delta}^{-}\right|}{\operatorname{Lip}(\varphi)}\right) \leq \theta\left(\left|u_{j}^{+}-u_{j}^{-}\right|\right)
$$

and together with (6.10) this implies

$$
\sup _{j} \int_{S_{u_{j, \delta}}}\left|u_{j, \delta}^{+}-u_{j, \delta}^{-}\right| d \mathcal{H}^{m-1} \leq \operatorname{Lip}(\varphi) M \frac{c \delta}{\theta(c \delta)} .
$$

In view of (6.9) it is possible to find numbers $\delta_{j}>0$, such that

$$
\delta_{j} \rightarrow 0 \text { and }\left\|\nabla u_{j, \delta_{j}}-\nabla u_{j}\right\|_{1 ; \Omega} \rightarrow 0 .
$$

By (6.8), (6.11) and (6.12) it follows that $u_{j, \delta_{j}} \in S B V\left(\Omega ; \mathbf{R}^{n}\right)$, that (6.5), (6.6) hold and that for any Borel set $A \subseteq \Omega$,

$$
\left|D u_{j, \delta_{j}}(A)\right| \leq\left|\int_{A} \nabla u_{j, \delta_{j}} d x\right|+\int_{A \cap S_{u_{j, \delta}}}\left|u_{j, \delta_{j}}^{+}-u_{j, \delta_{j}}^{-}\right| d \mathcal{H}^{m-1} \rightarrow 0,
$$

hence that (6.7) holds.

3. Define for each $j$ the non-negative, finite Radon measure

$$
\mu_{j}(A)=\int_{A}\left|\nabla u_{j, \delta_{j}}\right| d x+\int_{A \cap S_{u_{j, \delta}}}\left|u_{j, \delta_{j}}^{+}-u_{j, \delta_{j}}^{-}\right| d \mathcal{H}^{m-1}, A \in \mathbf{B}(\Omega) .
$$


Define for each $h \in \mathcal{C}_{0}^{0}(\Omega)$ the number

$$
\langle\mu ; h\rangle=\sum_{j=1}^{\infty} \frac{\left\langle\mu_{j} ; h\right\rangle}{2^{j}\left(1+\mu_{j}(\Omega)\right)} .
$$

It is readily seen that $\mu$ hereby is a bounded, linear functional on $\mathcal{C}_{0}^{0}(\Omega)$ and that $\langle\mu ; h\rangle \geq 0$ if $h \geq 0$. Therefore $\mu$ is a non-negative, finite Radon measure on $\Omega$.

From the definition it follows that each $D u_{j, \delta_{j}}$ is $\mu$-AC. Let $A$ be a $\mu$ measurable subset of $\Omega$. Since Radon measures are Borel regular there is a Borel subset $B$ of $\Omega$, such that $A \subseteq B$ and $\mu(B \backslash A)=0$. By $\mu$-AC and (6.7)

$$
D u_{j, \delta_{j}}(A)=D u_{j, \delta_{j}}(B) \rightarrow 0,
$$

and hence by the Vitali-Hahn-Saks Theorem $\left\{D u_{j, \delta_{j}}\right\}$ is uniformly $\mu$-AC. The conclusion now follows from Theorem 1.3.

Proof of Theorem 1.2. The proof is analogous to the proof for Theorem 1.1 and is omitted here. Notice also that it is possible to state the result in terms of $G S B V$-functions as mentioned in the remark following Theorem 1.2.

\section{$7 \quad$ Examples and remarks}

We discuss the various hypotheses encountered in the paper and give examples showing that some are indispensable.

Ad. (H1). The condition that $F$ is a normal integrand does not appear to be necessary for Theorems 1.1 and 1.2 to hold. Indeed, in the case $n=1$ much less is needed if the integrand is autonomous as is shown in [27]. However, for the method employed in this paper it seems that being a normal integrand is close to the weakest possible regularity assumption on $F$.

Under the assumption that $F$ is a non-negative Carathéodory integrand satisfying the growth condition $\left(\mathrm{H} 3_{p}\right)$, the result of [1] states that quasiconvexity of $F(x, v, X)$ in $X$ for almost all $x$ and all $v$ is necessary and sufficient for $I(u)$ to be sequentially weakly lower semicontinuous on $W^{1, p}$ (and similarly for $p=\infty$ ). So far no necessary condition has been found if $F$ is merely assumed to be a normal integrand. The condition should be related to quasiconvexity, but it is likely that it also involves the $v$-variable.

Ad. (H2). Quasiconvexity is the natural assumption in the multi-dimensional case. It is however very hard to verify that a given function is quasiconvex and partly for this reason rank-1 convexity and polyconvexity have been studied in the calculus of variations. The function $f: \mathbf{R}^{n \times m} \rightarrow \mathbf{R} \cup\{ \pm \infty\}$ is rank-1 convex if it is convex on rank-1 lines in $\mathbf{R}^{n \times m}$ and it is polyconvex if $f(X)$ is a convex function of the minors of $X$ (e.g. if $m=n=2, f$ is polyconvex if $f(X)=h(X, \operatorname{det} X)$, where $h$ is convex).

If $f$ is $\mathcal{C}^{2}$, then rank-1 convexity of $f$ is equivalent to the Legendre-Hadamard condition for $\nabla^{2} f: \quad \nabla^{2} f(X)(a \otimes b, a \otimes b) \geq 0 \quad \forall X, a, b$. The notion of polyconvexity was introduced by Ball in [12] (some special cases appear implicit in [43], [44]) and is related to null 
Lagrangians and to weak sequential continuity (see also [14] and [21]). For real-valued functions we have schematically:

$$
\text { polyconvex } \Rightarrow \text { quasiconvex } \Rightarrow \text { rank-1 convex. }
$$

If $\min \{m, n\}=1$, then the concepts reduce to ordinary convexity, whereas for $m, n>1$ there are quadratic polyconvex functions that are not convex. For $m, n>1$ there exist quasiconvex functions that are not polyconvex (see [45] and the references therein). Rank1 convexity is equivalent to quasiconvexity for quadratic forms in all dimensions and if $\min \{m, n\}=2$, then it is even equivalent to polyconvexity (see [21] and the references therein). It is not difficult to show that the same is true for polynomials of at most third degree. However, as shown by Šverák in [55], there are polynomials of degree 4 on $\mathbf{R}^{n \times m}$, which are rank- 1 convex but not quasiconvex when $n \geq 3, m \geq 2$. This example confirmed a conjecture from [43] (for dimensions $n \geq 3, m \geq 2$ ). Morrey's conjecture, that rank-1 convexity does not imply quasiconvexity, is still open in dimensions $n=2, m \geq 2$. We refer to [45] for a further discussion of the matter.

Ad. $\left(\mathrm{H} 3_{p}\right)$. The lower semicontinuity results fail without the growth condition $\left(\mathrm{H} 3_{p}\right)$ (cf. [15]). In [15] Ball and Murat observed that in the case, where $F(x, v, X)=f(X)$ is bounded from below (but allowed to be $\infty$ ) a necessary condition for $I(u)$ to be sequentially weakly lower semicontinuous on $W^{1, p}$ is that the condition (2.8) holds for all $u \in W_{0}^{1, p}\left(\omega ; \mathbf{R}^{n}\right)$. They called this strengthening of the ordinary quasiconvexity condition for $W^{1, p}$-quasiconvexity (ordinary quasiconvexity corresponds to $W^{1, \infty}$-quasiconvexity). It is not hard to see that if $F(x, v, X)=f(X)$ satisfies $\left(\mathrm{H} 3_{p}\right)$, then quasiconvexity is equivalent to $W^{1, p}$-quasiconvexity. The $W^{1, p}$-quasiconvexity condition depends in a dramatic way on $p$. As a consequence of [15] Theorem 4.1, it follows that for $m=n \geq 2$ the function $f(X)=|\operatorname{det} X|$ is $W^{1, p}$-quasiconvex if and only if $p \geq m$ (see also [28]). It is still an open question whether $W^{1, p}$-quasiconvexity together with some regularity of $f$, e.g. continuity, is sufficient for sequential weak lower semicontinuity of $I$ on $W^{1, p}$ too. We notice that by Example 3.5 of [15] there are lower semicontinuous functions $f$, which are $W^{1,1}$ quasiconvex, but not rank-1 convex. A result of Tartar ([57], p. 164) states, also in this generality, that rank-1 convexity is a necessary condition for sequential weak lower semicontinuity of $I$. Hence some additional assumption is needed in general for $W^{1, p}$-quasiconvexity to be a sufficient condition for sequential weak lower semicontinuity on $W^{1, p}$. See [15], Theorem 3.1 and Conjecture 3.7 and the remarks afterwards. Partial results have been obtained in [38] and [35].

In [47] Pedregal observed that if the $W^{1, p}$-quasiconvexity condition is slightly strengthened it becomes sufficient. Following Pedregal a Borel function $f: \mathbf{R}^{n \times m} \rightarrow \mathbf{R} \cup\{\infty\}$, which is bounded from below is closed $W^{1, p}$-quasiconvex if for all probability measures $\nu$ on $\mathbf{R}^{n \times m}$ for which $\left(\mathcal{L}^{m}\lfloor\Omega) \otimes \nu\right.$ is a gradient $p$-Young measure, Jensen's inequality holds for $f$ and $\nu$ :

$$
\int f d \nu \geq f(\bar{\nu}), \text { where } \bar{\nu}=\int X d \nu(X)
$$

Whether this condition is necessary for $I$ to be sequentially weakly lower semicontinuous on $W^{1, p}$ too is still an open problem. Note that under the growth condition $\left(\mathrm{H} 3_{p}\right)$ quasiconvexity is equivalent to closed $W^{1, p}$-quasiconvexity and that the results in Theorems 1.1 and 1.2 remain valid if instead of $(\mathrm{H} 2)$ and $\left(\mathrm{H} 3_{p}\right)$ we assume that $F(x, v, X)$ is 
closed $W^{1, p}$-quasiconvex in $X$. Notice also that with this assumption $F=\infty$ is allowed. An example in [17] shows that the structure of gradient $p$-Young measures can be rather complicated. Consequently, the question of whether, for sufficiently regular functions, $W^{1, p}$-quasiconvexity is equivalent to closed $W^{1, p}$-quasiconvexity might be subtle. Partial results in this direction have been obtained in [35].

The next result shows the significance of $\left(\mathrm{H}_{p}\right)$ for multiple integrals $I$ with integrands $F(x, v, X)=f(X)$. The result is well-known and is a special case of a general result due to Alberti [4]. Our proof is elementary and cannot be adapted to treat the general case considered in [4].

Let $f: \mathbf{R}^{n \times m} \rightarrow \mathbf{R} \cup\{\infty\}$ be a lower semicontinuous function, which is bounded from below. Let $p \in[1, \infty), u_{0} \in W^{1, p}\left(\Omega ; \mathbf{R}^{n}\right)$ and define

$$
\begin{gathered}
\mathcal{A}=\left\{u \in W^{1, p}\left(\Omega ; \mathbf{R}^{n}\right): u-u_{0} \in W_{0}^{1, p}\left(\Omega ; \mathbf{R}^{n}\right)\right\}, \\
I(u)=\int_{\Omega} f(\nabla u) d x .
\end{gathered}
$$

Proposition 7.1 The functional $I$ is finite on $\mathcal{A}$ if and only if there exists a constant $c>0$, such that

$$
\forall X \in \mathbf{R}^{n \times m}: \quad f(X) \leq c\left(1+|X|^{p}\right) .
$$

Remark. Instead of requiring $I<\infty$ on $\mathcal{A}$ it is enough if $\{u \in \mathcal{A}: I(u)<\infty\}$ has an interior point in $\mathcal{A} \subset W^{1, p}\left(\Omega ; \mathbf{R}^{n}\right)$.

Proof. The 'if' part is trivial. Assume that $I<\infty$ on $\mathcal{A}$ and consider $\mathcal{A} \subset W^{1, p}\left(\Omega ; \mathbf{R}^{n}\right)$ as a complete metric space. Without loss in generality we can assume that $f \geq 0$. By Fatou's Lemma $I$ is lower semicontinuous on $\mathcal{A}$. Since

$$
\bigcup_{t=1}^{\infty}\{u \in \mathcal{A}: I(u) \leq t\}=\mathcal{A}
$$

it follows from Baire's Theorem that for some $t$ the sub-level set $\{u \in \mathcal{A}: I(u) \leq t\}$ has non-empty interior. Assume that for $u_{1} \in \mathcal{A}$ and $\delta>0$

$$
I(u) \leq t
$$

holds whenever $u \in \mathcal{A}$ and $\left\|u-u_{1}\right\|_{1, p ; \Omega} \leq \delta$.

We next construct a test function $u$, which together with (7.1) yields the conclusion. Define $\eta:[0, \infty) \rightarrow[0,1]$ as $\eta(s)=1$ if $s \leq 1 / 2$ and $\eta(s)=(2-2 s)^{+}$if $s \geq 1 / 2$. Take $x_{0} \in \Omega$ to be a $p$-Lebesgue point of $\nabla u_{1}$ and consider $r \in\left(0, \operatorname{dist}\left(x_{0}, \partial \Omega\right)\right)$. For $X \in \mathbf{R}^{n \times m}$ define

$$
v^{r}(x)=\eta\left(\left|\frac{x-x_{0}}{r}\right|\right)\left(X\left(x-x_{0}\right)-u_{1}(x)\right), x \in \mathbf{R}^{m}
$$

and

$$
u^{r}=v^{r}+u_{1} \in \mathcal{A}
$$


Notice that for some $c_{1}=c_{1}(m, n, p)$

$$
\left\|u^{r}-u_{1}\right\|_{1, p ; \Omega}=\left\|v^{r}\right\|_{1, p ; \Omega} \leq c_{1}\left(|X| r^{m / p}+\left\|\nabla u_{1}\right\|_{p ; B_{x_{0}, r}}\right)
$$

for $r \in\left(0, \operatorname{dist}\left(x_{0}, \partial \Omega\right)\right)$.

Fix $c_{2}>c_{1}\left|\nabla u_{1}\left(x_{0}\right)\right|$ and take $R \in\left(0, \operatorname{dist}\left(x_{0}, \partial \Omega\right)\right)$, such that

$$
\left\|u^{r}-u_{1}\right\|_{1, p ; \Omega} \leq c_{2}(1+|X|) r^{m / p}
$$

if $r \in(0, R]$. Clearly,

$$
I\left(u^{r}\right) \geq \int_{B_{x_{0}, r / 2}} f\left(\nabla u^{r}\right) d x=\mathcal{L}^{m}\left(B_{x_{0}, r / 2}\right) f(X)
$$

and hence taking

$$
r=\min \left\{R,\left(\frac{\delta}{c_{2}(1+|X|)}\right)^{p / m}\right\}
$$

we infer from (7.1) that

$$
t \geq \mathcal{L}^{m}\left(B_{0,1 / 2}\right) \min \left\{R^{m},\left(\frac{\delta}{c_{2}(1+|X|)}\right)^{p}\right\} f(X)
$$

or

$$
f(X) \leq c\left(1+|X|^{p}\right)
$$

for some suitable $c$, which is independent of $X$.

Recall that any convex function $f: \mathbf{R}^{n \times m} \rightarrow \mathbf{R}$ is the point-wise limit of an increasing sequence of convex functions of linear growth at infinity. The results in [15] imply that a similar statement is false for quasiconvex functions. The next example shows that the situation does not improve if we relax the requirement and only try to approximate with rank-1 convex functions. Before turning to the details of this we first observe a simple consequence of a result due to Sivaloganathan [54] (see also [16]).

Let $\rho>0$ and $B_{0, \rho}=\left\{x \in \mathbf{R}^{m}:|x|<\rho\right\}$. A function $u: B_{0, \rho} \rightarrow \mathbf{R}^{m}$ is called radial if there exists a function $R:[0, \rho] \rightarrow \mathbf{R}$, such that for almost all $x$

$$
u(x)=\frac{R(|x|)}{|x|} x .
$$

Recall (see e.g. [15]) that for $m>1$ and $p \geq 1$ the radial function $u$ belongs to $W^{1, p}\left(B_{0, \rho} ; \mathbf{R}^{m}\right)$ if and only if $R:(0, \rho) \rightarrow \mathbf{R}$ can be taken absolutely continuous and such that

$$
\int_{0}^{\rho} r^{m-1}\left(\left|R^{\prime}(r)\right|^{p}+\left|\frac{R(r)}{r}\right|^{p}\right) d r<\infty .
$$

Proposition 7.2 Let $f: \mathbf{R}^{m \times m} \rightarrow \mathbf{R}$ be a rank-1 convex function, which for some $p \geq 1$ satisfies the growth condition

$$
\limsup _{X \rightarrow \infty} \frac{|f(X)|}{|X|^{p}}<\infty
$$


Then for $X \in \mathbf{R}^{m \times m}$ and radial functions $u \in W_{0}^{1, p}\left(B ; \mathbf{R}^{m}\right)$ the inequality

$$
f_{B} f(X+\nabla u) d x \geq f(X)
$$

holds, where $B$ denotes the open unit ball in $\mathbf{R}^{m}$.

Proof. We can assume that $X=0$. By [54] the inequality (7.4) holds if $u$ is a smooth radial function. Fix any radial function $u$ in $W_{0}^{1, p}\left(B ; \mathbf{R}^{m}\right)$ and take $\rho>1$. Using a standard mollifier argument we can find smooth radial functions $u_{j}: B_{0, \rho} \rightarrow \mathbf{R}^{m}$, where $u_{j}(x)=0$ if $|x|=\rho$, such that $\left\|u-u_{j}\right\|_{1, p ; B_{0}, \rho} \rightarrow 0$, where we have extended $u$ by 0 outside $B$. By the growth assumption (7.3)

$$
\int_{B_{0, \rho}} f\left(\nabla u_{j}\right) d x \rightarrow \int_{B_{0, \rho}} f(\nabla u) d x=\int_{B} f(\nabla u) d x+f(0) \mathcal{L}^{m}\left(B_{0, \rho} \backslash B\right)
$$

and since $u_{j}$ is a smooth, radial function vanishing at $\partial B_{0, \rho}$

$$
\int_{B_{0, \rho}} f\left(\nabla u_{j}\right) d x \geq f(0) \mathcal{L}^{m}\left(B_{0, \rho}\right)
$$

we deduce (7.4).

Proposition 7.3 Let $f: \mathbf{R}^{m \times m} \rightarrow \mathbf{R} \cup\{\infty\}$ be a function with the property that it can be approximated from below by rank-1 convex functions $f_{j}$, i.e., $f_{j} \leq f$ and $f_{j} \rightarrow f$ pointwise, each $f_{j}$ verifying the growth condition (7.3) for some fixed $p \geq 1$. Then (7.4) holds for $f$ and all radial functions $u \in W_{0}^{1, p}\left(B ; \mathbf{R}^{m}\right)$.

Proof. This is easy.

Example 7.4 Let $p \in[1,2)$ and define $f(X)=|X|^{p}+|\operatorname{det} X|, X \in \mathbf{R}^{2 \times 2}$. Then $f$ is polyconvex and satisfies the $p, 2$ growth condition

$$
|X|^{p} \leq f(X) \leq 2|X|^{2}+1
$$

We claim that $f$ cannot be approximated from below with rank-1 convex functions $f_{j}$ that grows polynomially slower at $\infty$ than $f$, i.e., for some $q<2$

$$
\limsup _{X \rightarrow \infty} \frac{\left|f_{j}(X)\right|}{|X|^{q}}<\infty
$$

holds for each $j$. The following argument is inspired by the proof of Theorem 4.1 in [15].

Reductio ad absurdum: assume that for some $q<2$ this is possible. Then by Proposition 7.3 the inequality (7.4) holds for $f$ and all radial functions $u \in W_{0}^{1, q}\left(B ; \mathbf{R}^{2}\right)$. However, this cannot be true. Indeed, for $\lambda>0$ introduce the radial function

$$
u_{\lambda}(x)=\frac{\sqrt{\lambda}(1-|x|)}{|x|} x, x \in B .
$$


Observe that $u_{\lambda} \in W_{0}^{1, q}\left(B ; \mathbf{R}^{2}\right)$ and therefore by the assumption and Proposition 7.3, taking $X=\sqrt{\lambda} I$ and $u=u_{\lambda}$ in (7.4)

$$
f_{B} f\left(\sqrt{\lambda} I+\nabla u_{\lambda}\right) d x \geq f(\sqrt{\lambda} I)
$$

Since $f(\sqrt{\lambda} I)=2^{p} \lambda^{p / 2}+\lambda$ and

$$
f_{B} f\left(\sqrt{\lambda} I+\nabla u_{\lambda}\right) d x \leq c_{p} \lambda^{p / 2}
$$

we obtain

$$
c_{p} \lambda^{p / 2} \geq 2^{p} \lambda^{p / 2}+\lambda
$$

Since $p<2$ this is a contradiction if $\lambda$ is large enough.

Ad. (1.5) and (1.12). That it is necessary to impose some additional condition on the sequence of negative parts follows from Counterexample 7.3 in [15]. There it is shown that if $m=n=2$ and $F(x, v, X)=\operatorname{det} X$, then $I(u)$ is not sequentially weakly lower semicontinuous on $W^{1,2}$. In [42] Meyers found a technical condition which might be relevant in this connection. However, no attempt has been made to investigate this condition in the present setting.

The content of the next example is well-known and shows, on the level of Young measures, the difference between the multi-dimensional case $m, n>1$ (considered in this paper) and the scalar case $\min \{m, n\}=1$.

Example 7.5 Let $p \in[1, \infty]$ and $\nu$ be a Young measure on $\Omega \times \mathbf{R}^{n \times m}$ with a finite $p^{t h}$ order moment. Assume that

$$
\nu=\int_{\Omega} \delta_{x} \otimes \nu_{x} d x \text { and } \bar{\nu}_{x}=0 \text { a.e. }
$$

If $m=1$ or $n=1$, then $\nu$ is a gradient $p$-Young measure. For $m, n \geq 2$ this is in general false.

(The first assertion follows because quasiconvexity is just convexity in the case $\min \{m, n\}=$ 1. The second assertion follows from the fact that if $m, n \geq 2$ there are quasiconvex functions, which are not convex. If $p \geq 2$ we can take any second order minor and if $p \in[1,2)$ the examples are provided in e.g. [60].)

The following example concerns the hypotheses (1.9) and (1.11) in Theorems 1.2 and 1.4 .

Example 7.6 Let $p \in[1, \infty]$ and $\nu$ be a Young measure on $\Omega \times \mathbf{R}^{n \times m}$ with a finite $p^{t h}$ order moment. Assume that

$$
\nu=\int_{\Omega} \delta_{x} \otimes \nu_{x} d x \text { and } \bar{\nu}_{x}=0 \text { a.e. }
$$


There exists a sequence $\left\{u_{j}\right\} \subset S B V\left(\Omega ; \mathbf{R}^{n}\right)$ with the properties

$$
\left\|u_{j}\right\|_{1 ; \Omega} \rightarrow 0, \sup _{j}\left(\left\|\nabla u_{j}\right\|_{p ; \Omega}+\int_{S_{u_{j}}}\left|u_{j}^{+}-u_{j}^{-}\right| d \mathcal{H}^{m-1}\right)<\infty,
$$

$\left\{\left|\nabla u_{j}\right|^{p}\right\}$ is uniformly summable on $\Omega$ if $p<\infty$

and

$$
\varepsilon_{\nabla u_{j}} \rightarrow \nu \text { weakly* }^{*} \text { in } \mathcal{C}_{0}^{0}\left(\Omega \times \mathbf{R}^{n \times m}\right)^{\prime} .
$$

Proof. For simplicity we assume that $\Omega=B=\left\{x \in \mathbf{R}^{m}:|x|<1\right\}$ and that $\nu$ is homogeneous: $\nu=\left(\mathcal{L}^{m}\lfloor B) \otimes \nu^{\prime}\right.$. We also assume that $p<\infty$; the case $p=\infty$ can be treated analogously.

There is a sequence $\left\{V_{j}\right\}$, such that $V_{j} \rightarrow 0$ weakly in $L^{p}\left(B ; \mathbf{R}^{n \times m}\right),\left\{\left|V_{j}\right|^{p}\right\}$ is uniformly summable on $B$ and $\left\{V_{j}\right\}$ generates $\nu$ (see [36]). It is not restrictive to assume that each $V_{j}$ is compactly supported in $B$. In view of Theorem 3 in [2] applied to each row of $V_{j}$ we can find $v_{j} \in S B V\left(B ; \mathbf{R}^{n}\right)$ with the properties

$$
\nabla v_{j}=V_{j} \text { and } \int_{S_{v_{j}}}\left|v_{j}^{+}-v_{j}^{-}\right| d \mathcal{H}^{m-1} \leq c\left\|V_{j}\right\|_{1 ; B},
$$

where $c$ is a constant depending on $m$ and $n$ only. Because $V_{j}$ is compactly supported in $B$ we can also take $v_{j}$ to be compactly supported in $B$.

Put $t_{j}=\left\|v_{j}\right\|_{1 ; B}$ and consider the family $\mathcal{F}_{j}$ of all closed balls of radius less than $\left(j t_{j}\right)^{-1}$, which are contained in $B$. By Vitali's Covering Theorem there exists an at most countable sub-family $F_{j}$, which covers $\mathcal{L}^{m}$-almost all of $B$ and consists of pairwise disjoint balls. Write $F_{j}=\left\{B_{x_{k}^{j}, r_{k}^{j}}\right\}_{k \in K_{j}}$ and define

$$
u_{j}(x)=r_{k}^{j} v_{j}\left(\frac{x-x_{k}^{j}}{r_{k}^{j}}\right) \text { if } x \in B_{x_{k}^{j}, r_{k}^{j}}, k \in K_{j} .
$$

Hereby $u_{j}$ is well-defined and it is readily verified that $u_{j} \in S B V\left(B ; \mathbf{R}^{n}\right)$, that

$$
\left\|u_{j}\right\|_{1 ; B} \leq 1 / j,\left|D u_{j}\right|(B) \leq\left|D v_{j}\right|(B) \leq(1+c)\left\|V_{j}\right\|_{1 ; B}
$$

and that $\left\{\left|\nabla u_{j}\right|^{p}\right\}$ is uniformly summable on $B$. Fix $\eta \in \mathcal{C}_{0}^{0}(B)$ and $\varphi \in \mathcal{C}_{0}^{0}\left(\mathbf{R}^{n \times m}\right)$ and compute

$$
\int_{B} \eta \varphi\left(\nabla u_{j}\right) d x=\int_{B}\left(\sum_{k \in K_{j}}\left(r_{k}^{j}\right)^{m} \eta\left(x_{k}^{j}+r_{k}^{j} x\right)\right) \varphi\left(V_{j}(x)\right) d x
$$

Since $\eta$ is uniformly continuous

$$
\sum_{k \in K_{j}}\left(r_{k}^{j}\right)^{m} \eta\left(x_{k}^{j}+r_{k}^{j} x\right) \rightarrow f_{B} \eta d y
$$

uniformly in $x \in B$ and consequently

$$
\int_{B} \eta \varphi\left(\nabla u_{j}\right) d x \rightarrow \int_{B} \eta d x \int \varphi d \nu^{\prime}
$$

The next example concerns the hypothesis (1.4) in Theorems 1.1 and 1.3. 
Example 7.7 Let $\nu$ be a Young measure on $\Omega \times \mathbf{R}^{n \times m}$ with a finite first order moment and assume that

$$
\nu=\int_{\Omega} \delta_{x} \otimes \nu_{x} d x \text { and } \bar{\nu}_{x}=0 \text { a.e. }
$$

There exists a sequence $\left\{u_{j}\right\} \subset \mathcal{C}_{c}^{1}\left(\Omega ; \mathbf{R}^{n}\right)$, such that

$$
\left\|u_{j}\right\|_{1 ; \Omega} \rightarrow 0, \sup _{j}\left\|\nabla u_{j}\right\|_{1 ; \Omega}<\infty
$$

and

$$
\varepsilon_{\nabla u_{j}} \rightarrow \nu \text { weakly* in } \mathcal{C}_{0}^{0}\left(\Omega \times \mathbf{R}^{n \times m}\right)^{\prime}
$$

Proof. As in Example 7.6, but instead of applying Theorem 3 of [2] we apply Theorem 1 with $p=1$ and $\varepsilon=1 / j$. We leave the details of this to the interested reader.

As mentioned in the Introduction there are sequences $\left\{u_{j}\right\}$ of functions satisfying the conditions of Theorems 1.1-1.4, but that do not allow a decomposition $\nabla u_{j}=\nabla v_{j}+E_{j}$, where $\left\{E_{j}\right\}$ converges strongly in $L^{1}$ and $\left\{v_{j}\right\}$ converges weakly in $W^{1,1}$. Before giving the example we state an auxiliary lemma.

It is well known that given any vector field $V$ of class $\mathcal{C}^{1}$ on $\mathbf{R}^{m}$, there exists a function whose gradient is $V$ if and only if $\operatorname{curl} V=0$, where $\operatorname{curl} V$ is the function of $\mathbf{R}^{m}$ into $\mathbf{R}^{m \times m}$ defined by

$$
(\operatorname{curl} V)_{r, s}=\frac{\partial V_{s}}{\partial x_{r}}-\frac{\partial V_{r}}{\partial x_{s}} \quad \text { for } r, s=1, \ldots, m .
$$

By a mollifier argument a similar result may be proved when $V$ is a distribution and $\operatorname{curl} V=0$ in the distributional sense.

Lemma 7.8 Let $\left\{V_{j}\right\}$ be a sequence converging weakly* to $V$ in $L^{\infty}\left(\mathbf{R}^{m} ; \mathbf{R}^{m}\right)$. If $V_{j}=$ $\nabla v_{j}+E_{j},\left\{E_{j}\right\}$ converges strongly in $L^{1}$ and $\left\{v_{j}\right\}$ converges weakly in $W^{1,1}$, then $\operatorname{curl} V_{j} \rightarrow$ curlV strongly in $W_{l o c}^{-1, p}$ for all $p<\infty$.

Here we recall that $h_{j} \rightarrow 0$ strongly in $W_{l o c}^{-1, p}$ means that for each open and bounded subset $\omega \subset \mathbf{R}^{m}$ and $p^{\prime}=p /(p-1)$ we have

$$
\left\langle h_{j} ; \varphi\right\rangle \rightarrow 0
$$

uniformly in $\varphi \in W_{0}^{1, p^{\prime}}(\omega)$ with $\|\varphi\|_{1, p^{\prime} ; \omega} \leq 1$.

Proof. Suppose that $v_{j} \rightarrow v$ weakly in $W^{1,1}$ and $E_{j} \rightarrow E$ strongly in $L^{1}$. Because $V=\nabla v+E$ and

$$
V_{j}-V=\nabla\left(v_{j}-v\right)+\left(E_{j}-E\right)
$$

we can without loss in generality assume that $V=E=0$ and $v=0$.

Let $t>2 M=2 \sup \left\|V_{j}\right\|_{\infty}$. Then we have that

$$
\int_{\left\{\left|\nabla v_{j}\right| \geq t\right\}}\left|\nabla v_{j}\right| d x \leq \frac{t}{t-M} \int_{\left\{\left|E_{j}\right| \geq t-M\right\}}\left|E_{j}\right| d x \rightarrow 0 .
$$


By Lemma 3.4 we may find a sequence $\left\{w_{j}\right\}$ which is bounded in $W^{1, \infty}\left(\mathbf{R}^{m}\right)$ and such that $v_{j}-w_{j} \rightarrow 0$ strongly in $W^{1,1}\left(\mathbf{R}^{m}\right)$. Since $E_{j}+\nabla v_{j}-\nabla w_{j}=V_{j}-\nabla w_{j} \rightarrow 0$ weakly* in $L^{\infty}$ and strongly in $L^{1}$ we have that $V_{j}-\nabla w_{j} \rightarrow 0$ strongly in $L_{l o c}^{p}$ for all $p<\infty$ and thus the claim of the lemma follows.

Example 7.9 Let $B$ denote the open unit ball in $\mathbf{R}^{m}$ and define for $u_{j} \in W^{1, \infty}(B)$ the functions $\bar{u}_{j} \in S B V\left(\mathbf{R}^{m}\right)$ as

$$
\bar{u}_{j}(x)=\left\{\begin{array}{cl}
u_{j}(x) & \text { if }|x|<1 \\
0 & \text { if }|x| \geq 1
\end{array}\right.
$$

If $u_{j} \rightarrow 0$ weakly* in $W^{1, \infty}(B)$, then $\bar{u}_{j} \rightarrow 0$ strongly in $L^{\infty}\left(\mathbf{R}^{m}\right)$,

$$
\mathcal{H}^{m-1}\left(S_{\bar{u}_{j}}\right) \leq \mathcal{H}^{m-1}(\partial B) \text { and }\left\{D \bar{u}_{j}\right\} \text { is uniformly } \mu \text {-AC, }
$$

where $\mu=\mathcal{L}^{m}\left\lfloor B+\mathcal{H}^{m-1}\left\lfloor\partial B\right.\right.$. However, in general $\left\{\operatorname{curl} \nabla \bar{u}_{j}\right\}$ does not converge strongly to 0 in $W_{l o c}^{-1, p}$ for $p>1$. Indeed, assume for simplicity that $m=2$ and let $\varphi \in \mathcal{C}_{c}^{\infty}\left(\mathbf{R}^{2}\right)$. Then

$$
\left\langle\frac{\partial \nabla \bar{u}_{j}}{\partial x}-\frac{\partial \nabla \bar{u}_{j}}{\partial y} ; \varphi\right\rangle=-\left\langle\frac{\partial D^{s} \bar{u}_{j}}{\partial x}-\frac{\partial D^{s} \bar{u}_{j}}{\partial y} ; \varphi\right\rangle=\int_{\partial B} u_{j}\left(\frac{\partial \varphi}{\partial x}-\frac{\partial \varphi}{\partial y}\right) d \mathcal{H}^{1}
$$

thus if $u_{j} \neq 0$ on $\partial B$, then for each $p^{\prime}<\infty$ we have that

$$
\sup \left|\left\langle\operatorname{curl} \nabla \bar{u}_{j} ; \varphi\right\rangle\right|=\infty,
$$

where the supremum is taken over, say, $\varphi \in \mathcal{C}_{c}^{\infty}\left(B_{0,2}\right)$ with $\|\varphi\|_{1, p^{\prime}} \leq 1$.

\section{Appendix}

This appendix contains a proof of the characterisation of gradient Young measures. The general result we set out to prove is the following, where we note that it differs from the results of Kinderlehrer and Pedregal [32,33] only in (c), where we test with rather special quasiconvex functions.

Theorem 8.1 Let $p \in[1, \infty]$ and let $\nu=\int_{\Omega} \delta_{x} \otimes \nu_{x} d x$ be a Young measure. Then $\nu$ is a gradient $p$-Young measure if the following three conditions are satisfied:

(a) $\nu$ has a finite $p^{\text {th }}$ order moment;

(b) there exists $u \in W^{1,1}\left(\Omega ; \mathbf{R}^{n}\right)$, such that $\bar{\nu}_{x}=\nabla u(x)$ almost everywhere;

(c) for all quasiconvex functions $f: \mathbf{R}^{n \times m} \rightarrow \mathbf{R}$ satisfying $f(X)=f^{\star \star}(X)=|X|$ for $|X|$ large the Jensen inequality

$$
\int f d \nu_{x} \geq f\left(\bar{\nu}_{x}\right)
$$

holds for almost all $x$. 
Conversely, if $\nu$ is a gradient $p$-Young measure, then there exists a $\mathcal{L}^{m}$ negligible set $N \subset \Omega$, such that for any quasiconvex function $f: \mathbf{R}^{n \times m} \rightarrow \mathbf{R}$ verifying the growth condition

$$
\limsup _{X \rightarrow \infty} \frac{f(X)}{|X|^{p}}<\infty, \quad \text { if } p<\infty
$$

and no growth condition if $p=\infty$, then the Jensen inequality

$$
\int f d \nu_{x} \geq f\left(\bar{\nu}_{x}\right)
$$

holds for all $x \in \Omega \backslash N$.

Some of the technical ingredients in the proof are taken from [34], however, the proof relies on the Hahn-Banach Separation Theorem and is in this sense similar in spirit to the original proofs in $[32,33]$. The key points distinguishing our proof are the use of Corollary 1.8 (or Proposition 1.10), the choice of function space (we treat the case of inhomogeneous Young measures directly) and the observation contained in Lemma 8.3.

We start with a lemma, which in the case $p=\infty$ is identical to Theorem 2.3 in [32]. The general result can be inferred from the results in [33], but we give a self-contained proof essentially following the strategy proposed in [32] for the case $p=\infty$.

Lemma 8.2 Let $p \in[1, \infty]$ and $B=\left\{x \in \mathbf{R}^{m}:|x|<1\right\}$. If $\nu$ is a gradient $p$-Young measure and $\nu=\int_{\Omega} \delta_{x} \otimes \nu_{x} d x$, then there is a $\mathcal{L}^{m}$ negligible set $N \subset \Omega$, such that for $x \in \Omega \backslash N$ the measure $\left(\mathcal{L}^{m}\lfloor B) \otimes \nu_{x}\right.$ is a gradient $p$-Young measure.

Proof. We only give the proof for the case $p \in(1, \infty)$. The remaining cases can be treated analogously. By assumption there exists a sequence $\left\{u_{j}\right\}$, such that for some $u \in W^{1, p}\left(\Omega ; \mathbf{R}^{n}\right)$

$$
u_{j} \rightarrow u \text { weakly in } W^{1, p}\left(\Omega ; \mathbf{R}^{n}\right)
$$

and

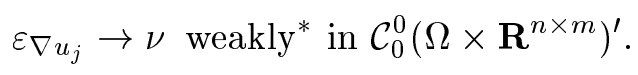

Extracting a subsequence if necessary we can assume that

$$
\left|\nabla u_{j}\right|^{p} \cdot \mathcal{L}^{m}\left\lfloor\Omega \rightarrow \mu \text { weakly* in } \mathcal{C}_{0}^{0}(\bar{\Omega})^{\prime},\right.
$$

where $\mu$ is a non-negative, finite Radon measure on the closure $\bar{\Omega}$ of $\Omega$. Let $\left\{\eta_{k}\right\}$ and $\left\{\varphi_{l}\right\}$ be two sequences, which are dense in $\mathcal{C}_{0}^{0}(B)$ and $\mathcal{C}_{0}^{0}\left(\mathbf{R}^{n \times m}\right)$, respectively. We can assume that each $\eta_{k} \in \mathcal{C}_{c}^{1}(B)$. Extend $\eta_{k}$ by 0 outside $B$ and put

$$
\bar{\varphi}_{l}(x)=\int \varphi_{l} d \nu_{x}
$$

In view of Theorem 2.4 we have for $x \in \Omega$ and $r<\operatorname{dist}(x, \partial \Omega)$

$$
\lim _{j \rightarrow \infty} \int_{\Omega} r^{-m} \eta_{k}\left(\frac{y-x}{r}\right) \varphi_{l}\left(\nabla u_{j}(y)\right) d y=\left(\check{\eta}_{k, r} \star \bar{\varphi}_{l}\right)(x)
$$


where $\check{\eta}_{k, r}(y)=r^{-m} \eta_{k}(-y / r)$ and $\star$ denotes convolution. Let $\Omega^{\prime}$ denote the set of points $x \in \Omega$, where

$$
\begin{aligned}
\lim _{r \rightarrow 0^{+}}\left(\check{\eta}_{k, r} \star \bar{\varphi}_{l}\right)(x) & =\int_{B} \eta_{k} d y \bar{\varphi}_{l}(x), \\
\lim _{r \rightarrow 0^{+}} \frac{\mu\left(\bar{B}_{x, r}\right)}{\mathcal{L}^{m}\left(B_{x, r}\right)} & =\frac{d \mu}{d \mathcal{L}^{m}}(x) \in \mathbf{R}
\end{aligned}
$$

and

$$
\lim _{r \rightarrow 0^{+}} r^{-p} f_{B_{x, r}}|u(y)-u(x)-\nabla u(x)(y-x)|^{p} d y=0 .
$$

Put $N=\Omega \backslash \Omega^{\prime}$ and notice that $\mathcal{L}^{m}(N)=0$. Fix $x \in \Omega \backslash N$ and introduce for $r \in$ $(0, \operatorname{dist}(x, \partial \Omega))$ the rescaled functions $u_{j}^{x, r}$ and $u^{x, r}$ as respectively

$$
u_{j}^{x, r}(y)=\frac{1}{r}\left(u_{j}(x+r y)-u(x)\right), y \in B
$$

and

$$
u^{x, r}(y)=\frac{1}{r}(u(x+r y)-u(x)), y \in B .
$$

For convenience of notation put

$$
\begin{gathered}
I_{i}(j, r)=\sum_{l+k \leq i}\left(\left|\int \eta_{k}(y) \varphi_{l}\left(\nabla u_{j}^{x, r}(y)\right) d y-\int_{B} \eta_{k} d y \bar{\varphi}_{l}(x)\right|\right)+ \\
+\int_{B}\left|u_{j}^{x, r}(y)-\nabla u(x) y\right|^{p} d y+\sup \left\{0, \int_{B}\left|\nabla u_{j}^{x, r}(y)\right|^{p} d y-\frac{d \mu}{d \mathcal{L}^{m}}(x)\right\}
\end{gathered}
$$

and notice that $\lim _{r \rightarrow 0^{+}} \lim _{j \rightarrow \infty} I_{i}(j, r)=0$ for each $i$. For each $i$ take first $r_{i} \in(0,1 / i)$, such that

$$
\lim _{j \rightarrow \infty} I_{i}\left(j, r_{i}\right)<1 / i
$$

and next $j_{i}>i$, so $I_{i}\left(j_{i}, r_{i}\right)<1 / i$. Define $v_{i}=u_{j_{i}}^{x, r_{i}}$ and notice that $\left\{v_{i}\right\}_{i=1}^{\infty} \subset W^{1, p}\left(B ; \mathbf{R}^{n}\right)$ has the properties

$$
v_{i} \rightarrow \nabla u(x)(\cdot) \text { weakly in } W^{1, p}\left(B ; \mathbf{R}^{n}\right)
$$

and

$$
\lim _{i \rightarrow \infty} \int_{B} \eta_{k} \varphi_{l}\left(\nabla v_{i}\right) d y=\int_{B} \eta_{k} d y \int \varphi_{l} d \nu_{x}
$$

for all $k, l=1,2, \ldots$ The proof is concluded by noticing that the linear span of tensor products $\eta_{k} \otimes \varphi_{l}$ is dense in $\mathcal{C}_{0}^{0}\left(B \times \mathbf{R}^{n \times m}\right)$.

Proof of the second part of Theorem 8.1. Apply Lemma 8.2 and Corollary 1.9.

In the remainder of this section we focus on proving the first part of Theorem 8.1. In view of Corollary 1.8 it suffices to show that $\nu$ is a gradient 1-Young measure. Since $\nu$ is a gradient 1-Young measure if and only if $\lambda=\int_{\Omega} \delta_{x} \otimes\left(\nu_{x} \star \delta_{-\nabla u(x)}\right) d x$ is and $\bar{\lambda}_{x}=0$ we can assume that $u \equiv 0$. Hence by Lemma 4.2 we can assume that the measure

$$
\nu=\int_{\Omega} \delta_{x} \otimes \nu_{x} d x
$$

satisfies the conditions: 
(a') $\int_{\Omega \times \mathbf{R}^{n \times m}}|X| d \nu(x, X)<\infty ;$

(b') $\bar{\nu}_{x}=0$ for almost all $x$;

(c') for all quasiconvex functions $f: \mathbf{R}^{n \times m} \rightarrow \mathbf{R}$ satisfying $f(X) /|X| \rightarrow 1$ as $X \rightarrow \infty$ the inequality

$$
\int f d \nu_{x} \geq f(0)
$$

holds for almost all $x$.

We introduce some notation for the proof. The space of Lipschitz functions $F: \Omega \times$ $\mathbf{R}^{n \times m} \rightarrow \mathbf{R}$ is a (non-separable) Banach space with the norm

$$
\|F\|=\left|F\left(x_{0}, 0\right)\right|+\operatorname{Lip}(F),
$$

where $x_{0} \in \Omega$ is arbitrary but fixed and $\operatorname{Lip}(F)$ denotes the Lipschitz constant of $F$. (The Lipschitz constant refers to the metric $\operatorname{dist}((x, X),(y, Y))=|x-y|+|X-Y|$.) Let $\mathcal{E}$ denote the subspace of Lipschitz functions $F: \Omega \times \mathbf{R}^{n \times m} \rightarrow \mathbf{R}$ with the property that the function

$$
(x, X) \mapsto \frac{F(x, X)}{1+|X|}
$$

admits a continuous extension to $\bar{\Omega} \times\left(\mathbf{R}^{n \times m} \cup\{\infty\}\right)$ (the closure of $\Omega$ times the one-point compactification of $\mathbf{R}^{n \times m}$ with the natural metric topology). It is readily verified that $\mathcal{E}$ is a closed subspace and hence that $(\mathcal{E},\|\cdot\|)$ is a Banach space. ( $\mathcal{E}$ is non-separable, but that is immaterial for our purposes.)

Let $\mathbf{P}$ denote the set of positive measures $\mu$ on $\Omega \times \mathbf{R}^{n \times m}$ satisfying

$$
\mu\left(A \times \mathbf{R}^{n \times m}\right)=\mathcal{L}^{m}(A) \text { for all Borel sets } A \subseteq \Omega \text { and } \int_{\Omega \times \mathbf{R}^{n \times m}}|X| d \mu(x, X)<\infty .
$$

Let $\mathbf{Y}$ denote the subset of $\mathbf{P}$ consisting of measures $\mu$ with the additional property that there exists a sequence $\left\{u_{j}\right\}$ in $W_{0}^{1,1}\left(\Omega ; \mathbf{R}^{n}\right)$, such that

$$
u_{j} \rightarrow 0 \text { weakly in } W_{0}^{1,1}\left(\Omega ; \mathbf{R}^{n}\right)
$$

and

$$
\varepsilon_{\nabla u_{j}} \rightarrow \mu \text { weakly* in } \mathcal{C}_{0}^{0}\left(\Omega \times \mathbf{R}^{n \times m}\right)^{\prime} .
$$

We regard $\mathbf{P}$ as a subset of the dual space $\mathcal{E}^{\prime}$ by the duality pairing

$$
\langle\mu ; F\rangle=\int F d \mu, F \in \mathcal{E},
$$

and hereby we have that

$$
\|\mu\|=\sup _{\|F\| \leq 1}\langle\mu ; F\rangle \leq c \int(1+|\cdot|) d \mu
$$

where $c>0$ is a constant depending on the diameter of $\Omega$ only.

We are going to show that $\nu \in \mathbf{Y}$ by using the Hahn-Banach Separation Theorem in the dual space of $\mathcal{E}$. Before proceeding to the details of this we need some auxiliary results. 
Lemma 8.3 If $\overline{\mathbf{Y}}$ denotes the weak ${ }^{*}$ closure of $\mathbf{Y}$ in $\mathcal{E}^{\prime}$, then $\overline{\mathbf{Y}} \cap \mathbf{P}=\mathbf{Y}$.

Proof. Fix $\xi \in \overline{\mathbf{Y}} \cap \mathbf{P}$. Take $\left\{\eta_{i}\right\}_{i=1}^{\infty} \subset W_{0}^{1, \infty}(\Omega)$, which is dense in $\mathcal{C}_{0}^{0}(\Omega)$ and $\left\{f_{j}\right\}_{j=1}^{\infty} \subset$ $W^{1, \infty}\left(\mathbf{R}^{n \times m}\right)$, which is dense in $\mathcal{C}_{0}^{0}\left(\mathbf{R}^{n \times m}\right)$. Define $F(x, X)=|X|,(x, X) \in \Omega \times \mathbf{R}^{n \times m}$, and notice that $F \in \mathcal{E}$. Since also $\eta_{i} \otimes f_{j}$ belongs to $\mathcal{E}$ for all $i, j \geq 1$ it follows that for each positive integer $k$ there exists $\nu_{k}$ in $\mathbf{Y}$, such that whenever $i+j \leq k$

$$
\left|\left\langle\nu_{k}-\xi ; F\right\rangle\right|+\left|\left\langle\nu_{k}-\xi ; \eta_{i} \otimes f_{j}\right\rangle\right|<\frac{1}{k}
$$

By definition of $\mathbf{Y}$ and in view of Theorem 2.4 we may take $u_{k} \in W_{0}^{1, \infty}\left(\Omega ; \mathbf{R}^{n}\right)$ verifying for $i+j \leq k$

$$
\int_{\Omega}\left|u_{k}\right| d x+\left|\int_{\Omega}\right| \nabla u_{k}\left|d x-\int_{\Omega \times \mathbf{R}^{n \times m}}\right| X\left|d \nu_{k}(x, X)\right|+\left|\int_{\Omega} \eta_{i} f_{j}\left(\nabla u_{k}\right) d x-\int \eta_{i} \otimes f_{j} d \nu_{k}\right|<\frac{1}{k} .
$$

Thus we have in particular for $i+j \leq k$

$$
\left|\int_{\Omega}\right| \nabla u_{k}\left|d x-\int_{\Omega \times \mathbf{R}^{n \times m}}\right| X|d \xi(x, X)|+\left|\int_{\Omega} \eta_{i} f_{j}\left(\nabla u_{k}\right) d x-\int \eta_{i} \otimes f_{j} d \xi\right|<\frac{2}{k},
$$

and therefore

$$
\lim _{k \rightarrow \infty} \int_{\Omega} \eta_{i} f_{j}\left(\nabla u_{k}\right) d x=\int \eta_{i} \otimes f_{j} d \xi
$$

for all integers $i, j \geq 1$ and

$$
\lim _{k \rightarrow \infty} \int_{\Omega}\left|\nabla u_{k}\right| d x=\int_{\Omega \times \mathbf{R}^{n \times m}}|X| d \xi(x, X) .
$$

We infer that

$$
\lim _{k \rightarrow \infty} \int_{\Omega} \eta f\left(\nabla u_{k}\right) d x=\int \eta \otimes f d \xi
$$

for all $(\eta, f) \in \mathcal{C}_{0}^{0}(\Omega) \times \mathcal{C}_{0}^{0}\left(\mathbf{R}^{n \times m}\right)$ and hence that $\left\{\nabla u_{k}\right\}$ generates the Young measure $\xi$. By (8.2) and Theorem 2.4, $\left\{\nabla u_{k}\right\}$ is uniformly summable and therefore $\left\{u_{k}\right\}$ converges weakly to 0 in $W^{1,1}\left(\Omega ; \mathbf{R}^{n}\right)$. This proves that $\xi \in \mathbf{Y}$.

The next result is an approximation result. It states that a general Young measure in $\mathbf{Y}$ can be approximated by piecewise constant Young measures from $\mathbf{Y}$. In the statement of the lemma we denote by $\mathcal{G}_{k}$ the collection of all open dyadic cubes $Q$ of side-length $2^{-k}$ contained in $\Omega$, that is

$$
\mathcal{G}_{k}=\left\{Q=2^{-k}\left(x+(0,1)^{m}\right) \subset \Omega: x \text { has integer coordinates }\right\}
$$

Lemma 8.4 Let $\xi=\int_{\Omega} \delta_{x} \otimes \xi_{x} d x \in \mathbf{Y}$ and let $k$ be a positive integer. If we define $\xi_{k} \in \mathbf{P}$ as

$$
\left\langle\xi_{k} ; \eta \otimes f\right\rangle=\sum_{Q \in \mathcal{G}_{k}} \int_{Q} \eta(x)\left(f_{Q} \int f d \xi_{y} d y\right) d x,
$$

for $\eta \in \mathcal{C}_{0}^{0}(\Omega), f \in \mathcal{C}_{0}^{0}\left(\mathbf{R}^{n \times m}\right)$, then $\xi_{k} \in \mathbf{Y}$ and $\xi_{k} \rightarrow \xi$ weakly* in $\mathcal{E}^{\prime}$. 
Proof. Since $\bar{\xi}_{x}=0$ almost everywhere in $\Omega$ we may apply the averaging principle (see e.g. Theorem 3.1 in [33] or [48]) to deduce that for each $Q \in \mathcal{G}_{k}$ the probability measure $\mu_{Q}$ defined as

$$
\left\langle\mu_{Q} ; f\right\rangle=f_{Q} \int f d \xi_{y} d y, f \in \mathcal{C}_{0}^{0}\left(\mathbf{R}^{n \times m}\right),
$$

gives rise to a homogeneous gradient 1-Young measure $\left(\mathcal{L}^{m}\lfloor Q) \otimes \mu_{Q}\right.$. Define $R_{k}=\Omega \backslash \bigcup \mathcal{G}_{k}$ and note that $\xi_{k}=\left(\mathcal{L}^{m}\left\lfloor R_{k}\right) \otimes \delta_{0}+\sum_{Q \in \mathcal{G}_{k}}\left(\mathcal{L}^{m}\lfloor Q) \otimes \mu_{Q}\right.\right.$; it follows from this that $\xi_{k} \in \mathbf{Y}$.

Considered as a linear functional on $\mathcal{E}$ the norm of $\xi_{k}$ is

$$
\left\|\xi_{k}\right\| \leq c \int_{\Omega} \int(1+|\cdot|) d \xi_{x} d x
$$

thus the sequence $\left\{\xi_{k}\right\}$ is norm-bounded in $\mathcal{E}^{\prime}$. To conclude the proof we fix $\eta \in W^{1, \infty}(\Omega)$, $f \in W^{1, \infty}\left(\mathbf{R}^{n \times m}\right)$, such that $\eta \otimes f \in \mathcal{E}$ and compute

$$
\left\langle\xi_{k} ; \eta \otimes f\right\rangle=\sum_{Q \in \mathcal{G}_{k}} \int_{Q} \eta(x) f_{Q} \int f d \xi_{y} d y d x .
$$

Because the function $x \mapsto \int f d \xi_{x}$ is summable it follows that $\left\langle\xi_{k} ; \eta \otimes f\right\rangle \rightarrow\langle\xi ; \eta \otimes f\rangle$ and the lemma follows from this.

Lemma 8.5 If $\overline{\mathrm{cO}} \mathbf{Y}$ denotes the weakly* closed convex hull of $\mathbf{Y}$ in $\mathcal{E}^{\prime}$, then $\overline{\mathrm{co}} \mathbf{Y} \cap \mathbf{P}=\mathbf{Y}$.

Proof. By Lemma 8.3 it suffices to prove that $\mathbf{Y}$ is convex. In view of Lemmata 8.3 and 8.4 it is enough to show that if $\mu_{1}=\left(\mathcal{L}^{m}\lfloor\Omega) \otimes \mu_{1}^{\prime}\right.$ and $\mu_{2}=\left(\mathcal{L}^{m}\lfloor\Omega) \otimes \mu_{2}^{\prime}\right.$ are two homogeneous measures in $\mathbf{Y}$, then also $\mu=t \mu_{1}+(1-t) \mu_{2}$ belongs to $\mathbf{Y}$ for each $t \in(0,1)$.

To start the proof we make the following observations. Let $U$ be a non-empty open, bounded subset of $\mathbf{R}^{m}$, let $u \in W_{0}^{1,1}\left(U ; \mathbf{R}^{n}\right)$ and define the probability measure $\nu_{u}^{\prime}$ as

$$
\left\langle\nu_{u}^{\prime} ; f\right\rangle=f_{U} f(\nabla u) d x, f \in \mathcal{C}_{0}^{0}\left(\mathbf{R}^{n \times m}\right)
$$

and $\nu_{u}=\left(\mathcal{L}^{m}\lfloor\Omega) \otimes \nu_{u}^{\prime}\right.$. Using the generalised Riemann-Lebesgue lemma (see e.g. [21], Theorem 1.5, p. 21) it is easy to show that $\nu_{u}$ belongs to $\mathbf{Y}$, and by Lemma 3.1 it is clear that any homogeneous measure in $\mathbf{Y}$ is the weak* limit in $\mathcal{E}^{\prime}$ of some sequence $\left\{\nu_{u_{j}}\right\}$, where $u_{j}$ belong to $W_{0}^{1,1}\left(\Omega ; \mathbf{R}^{n}\right)$.

Take open and bounded subsets $U_{1}, U_{2} \subset \mathbf{R}^{m}$ satisfying

$$
U_{1} \cap U_{2}=\varnothing \text { and } \mathcal{L}^{m}\left(U_{1}\right)=t \mathcal{L}^{m}\left(U_{1} \cup U_{2}\right) .
$$

Take $\left\{u_{i, j}\right\}_{j=1}^{\infty} \subset W_{0}^{1,1}\left(U_{i} ; \mathbf{R}^{n}\right)$, such that

$$
\lim _{j \rightarrow \infty}\left\langle\nu_{u_{i, j}} ; F\right\rangle=\left\langle\mu_{i} ; F\right\rangle, F \in \mathcal{E} ; i=1,2 .
$$

Define $v_{j} \in W_{0}^{1,1}\left(U_{1} \cup U_{2} ; \mathbf{R}^{n}\right)$ as

$$
v_{j}(x)= \begin{cases}u_{1, j}(x) & \text { if } x \in U_{1} \\ u_{2, j}(x) & \text { if } x \in U_{2}\end{cases}
$$


Clearly, $\nu_{v_{j}} \in \mathbf{Y}$ and for $F \in \mathcal{E}$

$$
\lim _{j \rightarrow \infty}\left\langle\nu_{v_{j}} ; F\right\rangle=\left\langle t \mu_{1}+(1-t) \mu_{2} ; F\right\rangle,
$$

hence $t \mu_{1}+(1-t) \mu_{2} \in \overline{\mathbf{Y}}$. The claim follows from Lemma 8.3.

Proof of first part of Theorem 8.1. In view of Lemma 8.5 and since $\nu \in \mathbf{P}$ it suffices to show that $\nu \in \overline{\mathrm{CO}} \mathbf{Y}$. In order to prove this we notice that by the Hahn-Banach Separation Theorem

$$
\overline{\mathrm{co}} \mathbf{Y}=\bigcap\{H: H \text { weakly* closed half-space containing } \mathbf{Y}\} \text {. }
$$

Let $H$ be a weakly* closed half-space in $\mathcal{E}^{\prime}$ that contains $\mathbf{Y}$. By definition there exists a weakly* continuous linear functional $T: \mathcal{E}^{\prime} \rightarrow \mathbf{R}$ and a number $t \in \mathbf{R}$, such that $H=\left\{l \in \mathcal{E}^{\prime}: T(l) \geq t\right\}$. A weakly* continuous linear functional is an evaluation functional, i.e. $T(l)=\langle l ; G\rangle, l \in \mathcal{E}^{\prime}$ for some $G \in \mathcal{E}$ (cf. [52]) and since $\mathbf{Y} \subset H$

$$
\langle\mu ; G\rangle \geq t
$$

for all $\mu \in \mathbf{Y}$. We claim that this implies that

$$
G^{q c} \in \mathcal{E} \text { and } \int_{\Omega} G^{q c}(x, 0) d x \geq t
$$

where for each $x \in \Omega, G^{q c}(x, \cdot)$ denotes the quasiconvex envelope of $G(x, \cdot)$.

By hypothesis (c') this entails that $\nu$ belongs to $H$. Hence we conclude the proof by verifying (8.3).

Recall that for a positive integer $k, \mathcal{G}_{k}$ denotes the family of all open dyadic cubes of side-length $2^{-k}$ contained in $\Omega$. For each $Q \in \mathcal{G}_{k}$ pick $u_{Q} \in W_{0}^{1,1}\left((0,1)^{m} ; \mathbf{R}^{n}\right)$ and extend each $u_{Q}$ to all of $\mathbf{R}^{m}$ by $(0,1)^{m}$ periodicity. Define $v_{j} \in W_{0}^{1,1}\left(\Omega ; \mathbf{R}^{n}\right)$ as

$$
v_{j}(x)=\left\{\begin{array}{cl}
\frac{1}{j 2^{k}} u_{Q}\left(j 2^{k} x\right) & \text { if } x \in Q ; Q \in \mathcal{G}_{k}, \\
0 & \text { otherwise. }
\end{array}\right.
$$

Then $v_{j} \rightarrow 0$ weakly in $W_{0}^{1,1}\left(\Omega ; \mathbf{R}^{n}\right)$ and $\left\{\nabla v_{j}\right\}$ generates the Young measure $\mu=\int_{\Omega} \delta_{x} \otimes$ $\mu_{x}^{\prime} d x$, where by use of the notation from the proof of Lemma 8.5 we have

$$
\mu_{x}^{\prime}=\left\{\begin{array}{cl}
\nu_{u_{Q}}^{\prime} & \text { if } x \in Q ; Q \in \mathcal{G}_{k} \\
\delta_{0} & \text { otherwise }
\end{array}\right.
$$

Clearly, $\mu \in \mathbf{Y}$ and so

$$
t \leq \int_{\Omega \backslash \cup \mathcal{G}_{k}} G(x, 0) d x+\sum_{Q \in \mathcal{G}_{k}} \int_{Q} \int_{(0,1)^{m}} G\left(x, \nabla u_{Q}(y)\right) d y d x .
$$

For each $Q \in \mathcal{G}_{k}$ we let $x_{Q} \in \Omega$ be the lower left corner point of $Q$. Since $G$ is Lipschitz continuous and the diameter of each $Q$ is $\sqrt{m} 2^{-k}$ we get

$$
t \leq \int_{\Omega \backslash \cup \mathcal{G}_{k}} G(x, 0) d x+\sqrt{m} 2^{-k} \operatorname{Lip}(G) \mathcal{L}^{m}(\Omega)+\sum_{Q \in \mathcal{G}_{k}} \mathcal{L}^{m}(Q) \int_{(0,1)^{m}} G\left(x_{Q}, \nabla u_{Q}(y)\right) d y
$$


or

$$
t-\varepsilon_{k} \leq \sum_{Q \in \mathcal{G}_{k}} \mathcal{L}^{m}(Q) \int_{(0,1)^{m}} G\left(x_{Q}, \nabla u_{Q}(y)\right) d y,
$$

where $\varepsilon_{k}>0$ is independent of the functions $u_{Q}$ and tends to 0 as $k$ tends to $\infty$. Taking the infimum over $u_{Q} \in W_{0}^{1,1}\left((0,1)^{m} ; \mathbf{R}^{n}\right)$ yields the inequality

$$
t-\varepsilon_{k} \leq \sum_{Q \in \mathcal{G}_{k}} \mathcal{L}^{m}(Q) G^{q c}\left(x_{Q}, 0\right)
$$

which is valid for each $k$. By Lemma 8.6 below it follows that $G^{q c}$ belongs to $\mathcal{E}$ and therefore we conclude the proof by letting $k$ tend to infinity and noticing that the right hand side is a Riemann sum for the integral $\int_{\Omega} G^{q c}(x, 0) d x$.

Lemma 8.6 Let $F \in \mathcal{E}$ and assume that there exists $(x, X) \in \Omega \times \mathbf{R}^{n \times m}$, such that $F^{q c}(x, X)>-\infty$. Then $F^{q c} \in \mathcal{E}$.

Proof. For $(x, X)$ and $(y, Y)$ we have

$$
F(x, X) \geq F(y, Y)-\operatorname{Lip}(F)(|x-y|+|X-Y|) .
$$

Taking $y=x_{0}$ and $Y=X$ yields

$$
F^{q c}(x, X) \geq F^{q c}\left(x_{0}, X\right)-\operatorname{Lip}(F)\left|x-x_{0}\right|,
$$

and therefore it follows that $F^{q c}$ is real-valued for all $(x, X)$. Next we take $Y=X+H$ and get

$$
F^{q c}(x, X) \geq F^{q c}(y, X+H)-\operatorname{Lip}(F)(|x-y|+|H|),
$$

whereby we conclude that $F^{q c}$ is Lipschitz continuous. It follows easily that $F^{q c}(x, X) /|X|$ has a finite limit as $X \rightarrow \infty$ and that this limit is independent of $x \in \Omega$.

\section{Acknowledgements}

It is a pleasure for me to record my thanks to G. Alberti, S. Demoulini, S. Müller and P. Plechác for stimulating discussions related to the subject of this paper. Parts of the work was carried out while the author was visiting the Max-Planck Institute for Mathematics in the Sciences, Leipzig. The support from the Danish Natural Science Research Council and from MPI is gratefully acknowledged.

\section{References}

[1] E. Acerbi and N. Fusco. Semicontinuity problems in the calculus of variations. Arch. Rat. Mech. Anal., 86:125-45, 1984.

[2] G. Alberti. A Luzin type property of gradients. J. Funct. Anal., 100:110-18, 1991.

[3] G. Alberti. Rank one properties for derivatives of functions with bounded variation. Proc. Roy. Soc. Edinburgh, 123A:239-74, 1993. 
[4] G. Alberti. Integral representation of local functionals. Ann. Mat. pura ed Appl., IV(CLXV):49-86, 1993.

[5] J.J. Alibert and G. Bouchitté. Non-uniform integrability and generalized Young measures. J. Convex Anal., 1997.

[6] L. Ambrosio. Existence theory for a new class of variational problems. Arch. Rat. Mech. Anal., 111:291-322, 1990.

[7] L. Ambrosio. On the lower semicontinuity of quasi-convex integrals in SBV. Nonlinear Analysis, 23(3):405-25, 1994.

[8] L. Ambrosio, G. Buttazzo and I. Fonseca. Lower semicontinuity problems in Sobolev spaces with respect to a measure. J. Math. Pures Appl., 75:211-24, 1996.

[9] L. Ambrosio and G. Dal Maso. On the relaxation in $B V\left(\Omega ; \mathbb{R}^{m}\right)$ of quasi-convex integrals. J. Funct. Anal., 109:76-97, 1992.

[10] L. Ambrosio and E. De Giorgi. Un nuovo tipo di funzionale del Calcolo delle Variazioni. Atti Accad. Naz. Lincei Cl. Sci. Fis. Mat. Nat., 82:199-210, 1988.

[11] E.J. Balder. A general approach to lower semicontinuity and lower closure in optimal control theory. SIAM J. Control and Optimization, 22(4):570-98, 1984.

[12] J.M. Ball. Convexity conditions and existence theorems in nonlinear elasticity. Arch. Rat. Mech. Anal., 63:337-403, 1977.

[13] J.M. Ball. A version of the fundamental theorem for Young measures. In PDE's and Continuum Models of Phase Transitions. Eds. M. Rascle, D. Serre, M. Slemrod. SpringerVerlag, Lecture Notes in Physics 344:207-15, 1989.

[14] J.M. Ball, J.C. Currie and P.J. Olver. Null Lagrangians, weak continuity, and variational problems of arbitrary order. J. Funct. Anal., 41:135-74, 1981.

[15] J.M. Ball and F. Murat. $W^{1, p}$ quasiconvexity and variational problems for multiple integrals. J. Funct. Anal., 58:225-53, 1984.

[16] J.M. Ball and F. Murat. Remarks on rank-one convexity and quasiconvexity. In Proc. 1990 Dundee Conference on Diff. Eq.

[17] J.M. Ball and K. Zhang. Lower semicontinuity of multiple integrals and the Biting Lemma. Proc. Roy. Soc. Edinburgh, 114A:367-79, 1990.

[18] H. Berliocchi and J.-M. Lasry. Intégrandes normales et mesures paramétrées en calcul des variations. Bull. soc. math. France, 101:129-84, 1973.

[19] G. Bouchitté, I. Fonseca and L. Mascarenhas. A global method for relaxation. Preprint 1997.

[20] N. Bourbaki. Intégration, Chap. IX, Éléments de Mathématique. Hermann Paris, 1969.

[21] B. Dacorogna. Direct Methods in the Calculus of Variations. Springer-Verlag 1989.

[22] N. Dunford and J.T. Schwartz. Linear operators, Vol. 1. Interscience 1967.

[23] L.C. Evans and R.F. Gariepy. Measure Theory and Fine Properties of Functions. Studies in advanced mathematics, CRC PRESS, 1992.

[24] I. Fonseca and S. Müller. Relaxation of Quasiconvex Functionals in $B V\left(\Omega ; \mathbb{R}^{p}\right)$ for Integrands $f(x, u, \nabla u)$. Arch. Rat. Mech. Anal., 123:1-49, 1993.

[25] I. Fonseca and S.Müller. A-quasiconvexity, lower semicontinuity and Young measures. Preprint 1998. 
[26] I. Fonseca, S. Müller and P. Pedregal. Analysis of concentration and oscillation effects generated by gradients. Preprint 1996.

[27] E. De Giorgi, G. Buttazzo and G. Dal Maso. On the lower semicontinuity of certain integral functionals. Atti Accad. Naz. Lincei., Cl. Sci. Fis. Mat. Natur., Rend., 74:274$82,1983$.

[28] P. Hajlasz. A note on weak approximation of minors. Ann. IHP Analyse Non Linéaire, 12(4):415-24, 1995.

[29] N. Hungerbühler. A refinement of Ball's theorem on Young measures. COCV 1997.

[30] T. Iwaniec and C. Sbordone. Weak minima of variational integrals, J. reine angew. Math., 454:143-61, 1994.

[31] A. Kalamajska. On lower semicontinuity of multiple integrals. Colloq. Math., 74(1):71-8, 1997.

[32] D. Kinderlehrer and P. Pedregal. Characterizations of Young measures generated by gradients. Arch. Rat. Mech. Anal., 115:329-65, 1991.

[33] D. Kinderlehrer and P. Pedregal. Gradient Young measures generated by sequences in Sobolev spaces. J. Geometric Anal., 4(1):59-90, 1994.

[34] J. Kristensen. Finite functionals and Young measures generated by gradients of Sobolev functions. Math. Inst., Technical University of Denmark, MAT-Report No. 1994-34, 1994.

[35] J. Kristensen. A necessary and sufficient condition for lower semicontinuity. Preprint.

[36] M. Kružík and T. Roubíček. Explicit characterization of $L^{p}$-Young measures. J. Math. Anal. Appl., 198:830-43, 1996.

[37] F.-C. Liu. A Luzin type property of Sobolev functions. Indiana Univ. Math. J., 26:645-51, 1977.

[38] J. Malý. Lower semicontinuity of quasiconvex integrals, Manuscripta Math., 85:419-28, 1994.

[39] J. Malý. Weak lower semicontinuity of polyconvex integrals. Proc. Royal Soc. Edinb., 123A:681-91, 1993.

[40] P. Marcellini. Approximation of quasiconvex functions and lower semicontinuity of multiple integrals. Manuscripta Math., 51:1-28, 1985.

[41] P. Marcellini. On the definition and the lower semicontinuity of certain quasiconvex integrals. Ann. I. H. P. Analyse Non Linéaire, 3:391-409, 1986.

[42] N.G. Meyers, Quasiconvexity and the semicontinuity of multiple variational integrals of any order. Trans. Amer. Math. Soc., 119:125-49, 1965.

[43] C.B. Morrey. Quasiconvexity and the semicontinuity of multiple integrals. Pacific J. Math., 2:25-53, 1952.

[44] C.B. Morrey. Multiple integrals in the Calculus of Variations. Springer-Verlag, 1966.

[45] S. Müller. Variational models for microstructure and phase transitions. Lectures at the C.I.M.E. summer school 'Calculus of variations and geometric evolution problems' Cetraro 1996.

[46] S. Müller. A sharp version of Zhang's theorem on truncating sequences of gradients. MaxPlanck-Institut für Mathematik in den Naturwissenschaften Leipzig, preprint no. 18, 1997. 
[47] P. Pedregal. Jensen's inequality in the calculus of variations. Differential and Integral Equations, 7(1):57-72, 1994.

[48] P. Pedregal. Parametrized Measures and Variational Principles. PNLDE 30, Birkhäuser, 1997.

[49] Y.G. Reshetnyak. General theorems on semicontinuity and on convergence with a functional. Siberian Math. J., 8:801-16, 1967.

[50] T. Roubíček. Relaxation in Optimization Theory and Variational Calculus. W. de Gruyter, 1997.

[51] W. Rudin. Real and Complex Analysis. McGraw-Hill, 1986.

[52] W. Rudin. Functional Analysis. Tata McGraw-Hill, New Delhi. 13th Reprint 1989.

[53] J. Serrin. A new definition of the integral for non-parametric problems in the calculus of variations. Acta. Math., 102:23-32, 1959.

[54] J. Sivaloganathan. Implications of rank one convexity. Ann. IHP Analyse Non Linéaire 5(2):99-118, 1988.

[55] V. Šverák. Rank-one convexity does not imply quasiconvexity. Proc. Royal Soc. Edinburgh, 120A:185-89, 1992.

[56] M. Sychev. Young measure approach to characterization of behaviour of integral functionals on weakly convergent sequences by means of their integrands. Preprint 1996.

[57] L. Tartar. Compensated compactness and applications to partial differential equations. In Nonlinear Analysis and Mechanics Heriot-Watt Symposium IV. Ed. Knops, R.J. Pitman Res. Notes in Math. 39, 1979.

[58] B. Yan. On the $L^{p}$-mean coercivity and relaxation of multiple integral functionals. Preprint 1996.

[59] L.C. Young. Generalized curves and the existence of an attained absolute minimum in the calculus of variations. Comptes Rendus de la Soc. des Sciences et de Lettres de Varsovie, classe III, 30:212-34, 1937.

[60] K. Zhang. A construction of quasiconvex functions with linear growth at infinity. Ann. Sc. Norm. Sup. Pisa Serie IV, XIX:313-26, 1992.

[61] W.P. Ziemer. Weakly Differentiable Functions. GTM 120, Springer-Verlag, 1989. 